Georgian Mathematical Journal

1(1994), No. 6, 599-640

\title{
POTENTIAL METHODS IN CONTINUUM MECHANICS
}

\author{
T. GEGELIA AND L. JENTSCH
}

\begin{abstract}
This is the survey of the applications of the potential methods to the problems of continuum mechanics. Historical review, new results, prospects of the development are given.
\end{abstract}

This survey paper is dedicated to the 90th birthday of Victor Kupradze. Therefore we shall cover here mainly questions connected with his scientific interests and dealt with by his pupils and followers. We wish to note specially that V. Kupradze's old works on the application of potential methods to the study of wave propagation, radiation and diffraction problems that had greatly contributed to the progress in these directions will hardly be mentioned.

Eight years have passed since our previous survey of the field in question. ${ }^{1}$ That was the period of great events in our life, change of the outlook, revaluation of many results, the arising of new difficulties in the development of science. The potential method keeps on developing and we do have results obtained in these years which are worthwhile being told about.

\section{A Historical Review}

1.1. Initiation of Potential Methods. When applied to problems of continuum mechanics, potential methods were initially based on the concept of representing solutions of these problems in the form of convolution type integrals, one of such convoluting functions being a special solution of the corresponding equation possessing a singularity and called the kernel of the potential. Later solutions of this kind came to be referred to as fundamental solutions, while convolution type integrals as potentials.

Potentials were constructed as early as the first half of the last century, proceeding from physical considerations. Another source for the construction of potentials was Green's formula (1828) and especially the representation of a regular function by means of this formula as the sum of a volume potential and single- and double-layer potentials. In the subsequent period

1991 Mathematics Subject Classification. 35Q, 73-02, 73C.

${ }^{1}$ See Burchuladze and Gegelia [1] where the reader can find sufficiently complete information on the development of the potential method in the elasticity theory.

599

1072-947X/1994/1100-0599\$07.00/0 (C) 1994 Plenum Publishing Corporation 
the investigation (Sobolev [1]) involved potential type integrals that were a combination of potentials of the above-mentioned three types:

$$
\mathcal{K}(\varphi)(x) \equiv \int_{X} K(x, x-y) \varphi(y) d \mu(y) .
$$

Here $X$ is some nonempty set from $\mathbb{R}^{m}, \mu$ is a complete measure over some class of subsets $X$ forming the $\sigma$-algebra, the kernel $K: Y \times \mathbb{R}^{m} \rightarrow \mathbb{C}$ ( $Y \subset X, \mathbb{C}$ is a set of complex numbers), the density $\varphi: X \rightarrow \mathbb{C}$. Thus the theory of a potential is the theory of an integral of type (1) dealing with the investigation of its boundary, differential and other properties. The potential method implies the application of a potential type integral to the study of problems of mathematical physics.

Alongside with methods of series, the potential methods have become a powerful tool of investigations in physics and mechanics. True, for some particulare domains methods of series gave both solutions of the problems and algorithms for the numerical realization of solutions, but for arbitrary domains the use of these methods was connected with certain difficulties. In this respect the method of the potential theory is undoubtedly more promissing. Moreover, algorithms provided by methods of series are not always convenient for numerical calculations, while potentials with integrals taken over the boundary of the considered medium, i.e., the so-called boundary integrals are very convenient for constructing numerical solutions. To this we should add that the prospect to represent solutions of problems of continuum mechanics by potentials in terms of boundary values and their derivatives looks very enticing. For a regular harmonic function, for example, such a representation formula immediately yields its analyticity, the character of its behaviour near singular points and other properties which are rather difficult to establish by the methods of series. Besides, the formula for representation of solutions in the form of potentials initiated the introduction of the Green function that had played an outstanding role in the development of the theory of boundary value problems.

1.2. Potentials of the Elasticity Theory. As it was mentioned in the foregoing subsection, kernels of potentials are constructed by special singular solutions of differential equations of problems under consideration. The construction of harmonic potentials is based on the fundamental solution of the Laplace equation. In other problems of mathematical physics use is made of fundamental and singular solutions of the corresponding differential equations. For example, in the elasticity theory potentials are constructed by means of the fundamental solution of the system of the basic equations of 
this theory. This system is written in terms of displacement components as

$$
\begin{gathered}
A\left(\partial_{x}\right) u=-F, \quad A\left(\partial_{x}\right)=\left\|A_{i j}\left(\partial_{x}\right)\right\|_{3 \times 3}, \\
A_{i j}\left(\partial_{x}\right) \equiv \delta_{i j} \mu \Delta+(\lambda+\mu) \frac{\partial^{2}}{\partial x_{i} \partial x_{j}}, \quad i, j=1,2,3,
\end{gathered}
$$

where $u=\left(u_{1}, u_{2}, u_{3}\right)$ is the displacement vector, $F$ is the volume force, $\lambda$ and $\mu$ are the Lamé constants, $\delta_{i j}$ is the Kronecker symbol, $\Delta$ is the Laplace operator. The fundamental solution of this system is the matrix (see, e.g., Kupradze, Gegelia, Basheleishvili and Burchuladze [1], which below will be referred to as Kupradze (1))

$$
\begin{gathered}
\Gamma(x)=\left\|\Gamma_{i j}(x)\right\|_{3 \times 3}, \quad \Gamma_{i j}(x) \equiv \frac{\lambda^{\prime} \delta_{i k}}{|x|}+\frac{\mu^{\prime} x_{i} x_{j}}{|x|^{3}} \\
\lambda^{\prime}=(\lambda+3 \mu)(4 \pi \mu(\lambda+2 \mu))^{-1}, \quad \mu^{\prime}=(\lambda+\mu)(4 \pi \mu(\lambda+2 \mu))^{-1} .
\end{gathered}
$$

whose each column (as well as each row) regarded as a vector satisfies the system (2) at any point of the space, except the origin, where this vector has the pole of first order.

This fundamental solution was constructed as far back as 1848 by the outstanding English physicist Lord Kelvin whose name at the time and till 1892 was Thomson. It was constructed proceeding from the physical arguments: if the entire space is filled up by an isotropic homogeneous elastic medium with the elastic Lamé constants $\lambda$ and $\mu$ and the unit concentrated force is applied to the origin, directed along the $x_{j}$-axis, then the displacement at the point $x$ produced by this force is equal to the $j$-th column of the matrix of fundamental solutions.

This result of Kelvin can hardly be overestimated. It had opened a vista for the potential method in the elasticity theory. Before long this discovery was followed by the works E. Betti, J. Boussinesq and others, where potentials of the elasticity theory were constructed and applied to boundary value problems.

The studies we have mentioned above belong mainly to the second half of the last century when the Fredholm theory did not exist. Therefore the potential methods were not applied to prove existence theorems of solutions of boundary value problems, and if they were, then there was no proper substantiation. From the results of that time we should draw the reader's attention to the solutions of numerous particular problems. Representatives of the Italian school were especially inclined to a wide use of potential methods (see the surveys Love [1], Tedone [1], Boussinesq [1], Trefftz [1], Marcolongo [1] and others).

The works of the scientists of the 19th century reflect an insufficient development of the mathematical means of that time. Mathematical arguments 
were largely based on physical considerations and proofs based on these considerations. Mathematicians of that time, including some oustanding ones, were quite content with the situation. For example, H. Poincaré wrote that one could not demand the same rigor of mechanics as of pure analysis. During a rapid development of the potential method suchlike opinions evidently led to the appearance of many statements having no mathematical substantiation. The theory of harmonic potentials, their boundary and differential properties had been developed only by the beginning of our century (H. Poincaré, O.D. Kellog, A.M. Liapunov, H.M. Günter, etc.), while the theory of potentials of elasticity in the second half of our century.

The fundamental solution of equations of fluid flow (the Stokes system) does not differ in any conspicuous way from the fundamental Kelvin matrix and the theory of the corresponding potentials is constructed similarly to potentials of the elasticity theory (see Lichtenstein [1], Odqvist [1], Ladyzhenskaya [1], Belonosov and Chernous [1]).

1.3. Invention of the Theory of Fredholm Integral Equations. The creation of the theory of integral equations by Fredholm gave a new impetus to the development of potential methods. In 1900 I. Fredholm proved his famous theorems for integral equations and the theorem of the existence of solution of the Dirichlet problem. The latter result made Fredholm worldwide famous and drew the attention of the mathematical community to the theory of integral equations. It was not difficult to guess what big prospects lay before Fredholm's discoveries - after all many problems of continuum mechanics are reduced by the potential method to integral equations. This formed the ground for the revival of potential methods and for a rapid development of the theory of integral equations (D. Hilbert, E. Goursat, G. Giraud, T. Carleman, F. Noether, E. Picard, H. Poincaré, J. Radon, F. Rellich, F. Riesz, F. Tricomi, E. Schmidt and many others).

Various problems of mathematical physics were reduced to various integral equations. In these problems the integration set was assumed to be a segment of the straight line, a finite or infinite domain from $\mathbb{R}^{m}$, a surface or a curve and so on. The resulting integral equations contained a continuous kernel, a kernel with a weak singularity, a symmetrical kernel and so on. In an attempt to cover general situations completely continuous operators were introduced and the foundations of functional analysis were laid (D. Hilbert, F. Riesz, S. Banach).

In investigating the Dirichlet problem, Fredholm sought for a solution in the form of a harmonic double-layer potential and obtained the integral equation. From the uniqueness of the solution of the Dirichlet problem he concluded that the corresponding homogeneous equation had only the trivial solution. In that case an alternative of his theory gave the theorem of the existence of solutions. However, Fredholm could not apply the same 
technique to the elasticity theory, since the double-layer potential of this theory leads to singular integral equations whose theory did not exist at his time. Using a roundabout way, namely, introducing the so-called pseudostress operator, in 1906 Fredholm succeeded in proving, by the potential method, the existence theorem of solution to the first basic problem of the elasticity theory.

This dicovery of Fredholm was no less important that the previous one. True, scientists had long been trying to prove the existence of solutions of the Dirichlet problems and their efforts had yielded positive results. Almost at the same time with I. Fredholm, H. Poincaré solved this problem using a different method (É. Picard, O. Perron). Poincaré's method is fit only for the Dirichlet problem for the Laplace equation and cannot be applied to the elasticity theory. This circumstance further enhanced interest in the potential method that previously was sometimes referred to as the Fredholm method but in recent years has come to be known as the method of boundary integral equations. The latter name reflects well the essence of the method from the standpoint of constructing numerical solutions, but the essence of the potential method is by no means confined to numerical analysis.

Though Fredholm's method was worthy of high praise, still it did not turn out to be universal. For example, it could not be applied to the investigation of the second problem of the elasticity theory. Scientists' efforts in this direction were vain (K. Korn, T. Boggio, H. Weyl, N. Kinoshita, T. Mura and others). They obtained singular integral equations for which Fredholm's theorems were not valid, while their attempts to introduce pseudostress analogues led to nothing. Neither were Fredholm's theorem valid for Wiener and Hopf's integral equations.

1.4. Singular Integral Equations. The theory of singular integral equations was developed only forty years after. In the 40ies this theory was worked out mainly by the Georgian mathematicians (see also the works by D. Hilbert, H. Poincaré, F. Noether and T. Carleman) led by N. Muskhelishvili but only for one-dimensional equations. It appeared that, unlike Fredholm's equations, the theory of singular equations largely depended on dimension of the integration set.

One-dimensional singular integral equations were fit for the investigation of only plane problems of mathematical physics. This initiated the era of a tempestuous development of plane problems. The situation was also facilitated by the well-developed theory of complex analysis connected, due to the efforts of N. Muskhelishvili, with plane problems of mechanics and one-dimensional singular integral equations (I. Vekua, N. Muskhelishvili, N. Vekua, D. Kveselava, D. Sherman, G. Mandzhavidze, M. Basheleishvili and others). 
1.5. Multidimensional Singular Integral Equations. It took another twenty years for the theory of multidimensional singular equations to acquire an ability to solve three-dimensional problems of mechanics. Three possible ways were available for constructing the theory of singular integral equations (SIE): it could be connected with the theory of complex analysis and boundary value problems of linear conjugation; it could be constructed by means of I. Vekua's inversion formulas and, finally, using the general theory of functional analysis. Only the third way is suitable for multidimensional SIE. But to apply methods of functional analysis one should have a conjugate equation in the sense of functional analysis, which cannot be done in Hölder spaces, as it is difficult to construct explicitly the conjugate space and to write the conjugate operator for these spaces. A formal application of the conjugate equation gives us nothing because it must be afterwards connected with the boundary value problem. N. Muskhelishvili managed to circumvent this difficulty by introducing the adjoint equation and proved the validity of Noether's theorems for this pair. In the multidimensional case SIE had to be investigated in the space $L_{2}$ (S. Mikhlin), and, after that, using the embedding theorems (T. Gegelia) in Hölder spaces. The Hölder space is necessary to obtain the classical solutions of problems of continuum mechanics.

The theory was elaborated sufficiently well in the 60ies mainly due to the efforts of S. Mikhlin and V. Kupradze (see also F. Tricomi, G. Giraud, T. Gegelia, A. Calderon, A. Zygmund, Gohberg [1], A.I. Volpert, Selley $[1,2]$ and others). By that time singular potentials had been studied completely (A. Calderon, A. Zygmund, Maz'ya [1], T. Gegelia and others) and the advantageous situation had formed for the application of potential methods. The results were not long in coming. The existence of solutions of the second basic problem of the elasticity theory (T.Gegelia, V. Kupradze), also of the third and the fourth problem (M. Basheleishvili, T. Gegelia) was proved. The dynamical problems of elasticity (V. Kupradze, T. Burchuladze, L. Magnaradze, T. Gegelia, O. Maisaia, R. Rukhadze, D. Natroshvili, R. Kapanadze, R. Chichinadze and others) and contact problems (V. Kupradze, M. Basheleishvili, T. Gegelia, Jentsch [5, 10, 14, 15], D. Natroshvili, M. Svanadze, R. Katamadze, R. Gachechiladze, M. Kvinikadze $[1,2]$, O. Maisaia and others) were studied completely. The improved models of an elastic medium were investigated, taking into account moment, heat and other stresses, electromagnetic and other fields (W. Nowacki, V. Kupradze, Jentsch [4, 8, 13], T. Burchuladze, M. Basheleishvili, D. Natroshvili, N. Kakhniashvili, T. Gegelia, T. Buchukuri, M. Agniashvili, Yu. Bezhuashvili, O. Napetvaridze, R. Gachechiladze, O. Maisaia, R. Chichinadze, R. Kapanadze, G. Javakhishvili, O. Jagmaidze, R. Dikhamindzhia, K. Svanadze, Zazashvili [1-3], R. Meladze, R. Rukhadze, Y. Adda, J. Philibert, J. Hlavaček, M. Hlavaček, J. Ignaczak, S. Kaliski, W. Nowacki and 
others).

The potential method was used to prove anew the theorems on the existence and uniqueness of solutions of plane problems and to investigate various two-dimensional models of the elasticity theory (M. Basheleishvili, G. Kvinikadze, Zh. Rukhadze, Jentsch [18-25], Jentsch and Maul [1], Zazashvili [2-4] and others).

1.6. Applications of Multidimensional SIE in the Elasticity Theory. Application of a newly created theory to applied problems usually demands serious intellectual effort, as well as a considerable amount of improvement and modific/ation of the theory itself. This is convincingly evidenced by the works starting from T. Carleman and F. Noether (1920-1923) and ending with N. Muskhelishivi (1945). The theory of one-dimensional SIE was developed mainly in the mentioned works by T. Carleman and F. Noether, but applications of the results stated therein began actually only after the publication of N. Muskhelishvili's monograph.

As compared with the one-dimensional case, the investigation of SIE in the multidimensional case was connected with difficulties of various nature. In the one-dimensional case all SIE are reduced to one and the same type of SIE with a Cauchy type kernel. However we do not have such a universal technique of representation for the multidimensional case. Here we deal with quite a variety of SIE characterized by the so-called SIE characteristic. Besides, the complicated topology connected with multidimensional SIE is yet another obstacle. Noether's theory holds for normal SIE in both the one-dimensional and the multidimensional case, but to verify the normality of one-dimensional SIE is not difficult at all, while in the multidimensional case the normality is established by means of the symbol matrix which is not always constructed explicitly. The calculation of the index becomes a much more difficult matter in the multidimensional case.

Naturally, the above-listed difficulties of the theory of multidimensional SIE complicate its application to problems of continuum mechanics. One has to seek for special techniques in order to establish the normality of the obtained SIE and to calculate their indices. Thus the theory of multidimensional SIE was created mainly in the 60ies but its improvement goes on to this day. The theory of SIE over open surfaces has not yet reached its perfection.

Let us illustrate what we have said above by the example of the classical elasticity theory.

1.7. Investigation of the Third Basic Problem of the Elasticity Theory. We shall consider the third boundary value problem of the classical elasticity theory. It consists in finding the solution $u=\left(u_{1}, u_{2}, u_{3}\right)$ of the system (2) in the domain $\Omega$ occupied by an elastic medium when tangential components of displacement and normal components of stress are given on 
the boundary $\partial \Omega$. The simplest technique for investigating this problem is to reduce it to the SIE system by means of the potential

$$
R(\varphi)(x)=\int_{\partial \Omega}\left(R\left(\partial_{y}, \nu\right) \Gamma(y-x)\right)^{*} \varphi(y) d_{y} S,
$$

where $\nu$ is the unit exterior normal vector to the surface $\partial \Omega$ at the point $y$, $\Gamma$ is the fundamental matrix (3), and

$$
\begin{gathered}
R\left(\partial_{y}, \nu\right)=\left\|R_{k j}\left(\partial_{y}, \nu\right)\right\|_{4 \times 3}, \\
R_{k j}\left(\partial_{y}, \nu\right)=\left(2 \mu \nu_{j} \frac{\partial}{\partial \nu}+\lambda \frac{\partial}{\partial x_{j}}\right) \delta_{k 4}+\left(\delta_{k j}-\nu_{k} \nu_{j}\right)\left(1-\delta_{k 4}\right) .
\end{gathered}
$$

As a result, for defining the uknown density $\varphi=\left(\varphi_{1}, \varphi_{2}, \varphi_{3}, \varphi_{4}\right)$ we obtain a rather complicated SIE system consisting of four equations for defining the three-component vector $u$.

The SIE theory elaborated, for example, in the monograph by S. Mikhlin cannot be applied directly to the obtained system. Therefore a nonstandard technique had to be developed in order to study the obtained SIE system (see Basheleishvili and Gegelia [2], Kupradze (1)). The application of this method of investigation of problems of the mentioned type to other models of continuum mechanics turned out to be a difficult matter that has not been coped with to the end.

\section{New Results. Prospects of the Development}

2.1. Basic Problems of the Elasticity Theory for an Anisotropic Medium. If the medium under consideration is an anisotropic one, then the investigation of boundary value problems becomes rather sophisticated for many reasons, for example, because in that case we do not have the corresponding fundamental matrix written explicitly in terms of elementary functions but for one exception (E. Kröner). It is given in the form

$$
\phi(x-y)=\Delta\left(\partial_{x}\right) \int_{B(0,1)}|(x-y) \cdot z| A^{-1}(z) d_{z} S,
$$

where $B(0,1)$ is the ball in $\mathbb{R}^{3}$ with center at the origin and radius equal to unity,

$$
A\left(\partial_{x}\right)=\left\|A_{i k}\left(\partial_{x}\right)\right\|_{3 \times 3}, \quad A_{i k}\left(\partial_{x}\right)=a_{i j k l} \frac{\partial^{2}}{\partial x_{j} \partial x_{l}},
$$

is the differential operator of the classical elaticity theory, $A^{-1}(z)$ is the reciprocal matrix to $A(z), \Delta$ is the Laplace operator, $a_{i j k l}$ are the elastic constants. Here and in what follows the summation over repeated indices is meant. 
The fundamental solution (5) was used as a basis for the elaboration of the potential theory (T. Gegelia, R. Kapanadze, Burchuladze and Gegelia [1]) by means of which boundary value problems were reduced to SIE systems. The main difficulty, however, is connected with the investigation of the obtained systems. The general SIE theory states that if the determinant of the symbol matrix of this system is different from zero everywhere, then the Noether theorems hold for SIE. As distinct from the isotropic case, the symbol matrix cannot be constructed effectively. R. Kapanadze succeeded in finding a beautiful way to overcome all obstacles. He connected, in some sense, the symbol matrices of the obtained SIE with the Cauchy problems for the definite simple systems of ordinary differential equations and proved the following theorem.

Theorem 1. The symbol determinants of SIE systems of boundary value problems are different from zero if and only if the corresponding homogeneous Cauchy problems have only trivial solutions.

The Cauchy problems have only trivial solutions under one natural restriction, namely under the positive definiteness of the specific energy of strain. This beautiful discovery of R. Kapanadze was used to investigate all the basic and contact problems of the classical elasticity theory for anisotropic media (see Kapanadze [1], Burchuladze and Gegelia [1], M. Basheleishvili, D. Natroshvili). Note that in investigation of the basic and the contact problems for an anisotropic homogeneous medium, i.e., when coefficients of the basic equations are constant numbers, the obtained singular integrals still depend on the pole. This is due to the fact that these integrals include derivatives of the fundamental matrix. If, however, the medium is anisotropic and nonhomogeneous, then the dependence of singular integrals on the pole is also due to the variability of equation coefficients. The method proposed by R. Kapanadze turns out suitable for this difficult situation, too. Moreover, R. Kapanadze showed that the above-mentioned connection of the boundary value problems with the corresponding Cauchy problems remains valid provided that the system under consideration is the strongly elliptic one. He thereby extended his method to the investigation of boundary value problems of couple-stress elasticity, thermoelasticity and other generalized models of an elastic anisotropic nonhomogeneous medium.

2.2. New Uniqueness Theorems for Problems of Statics. The uniqueness theorems of problems of the classical elasticity theory are treated in the fine monograph Knops and Payne [1], also in the book Kupradze (1) where the uniqueness theorems are also proved for couple-stress elasticity and thermoelasticity. The results of these monographs were afterwards improved and generalized to other models of an elastic medium (see Burchuladze and Gegelia [1]). 
Let an elastic isotropic homogeneous medium with the Lamé constants $\lambda$ and $\mu$ occupy the infinite domain $\Omega^{-}$which is a complement to the bounded domain $\Omega^{+}: \Omega^{-} \equiv \mathbb{R}^{3} \backslash \bar{\Omega}^{+}$. Then, under the assumptions of the classical theory, the static state of this medium is described by the system of equations (2). The following uniqueness theorem is proved (see Buchukuri and Gegelia [1-4]).

Theorem 2. Any basic problem of the static state of an elastic medium for the domain $\Omega^{-}$cannot have two regular solutions satisfying the condition

$$
u(x)=o(1)
$$

in a neighbourhood of infinity.

Note that in the classical uniqueness theorems (see Knops and Payne [1], Kupradze (1)), in addition to the condition (7), it is required that the decay condition at infinity

$$
\frac{\partial u(x)}{\partial x_{i}}=O\left(\frac{1}{|x|^{2}}\right), \quad i=1,2,3
$$

be fulfilled.

Theorem 2 was later on proved for anisotropic media (Buchukuri and Gegelia [3]), for problems of thermoelasticity, couple-stress elasticity (Buchukuri and Gegelia [4], a microporous elastic medium (Gegelia and Jentsch [1]).

In the second basic problem boundary stress vector is given on the boundary $\partial \Omega^{-}$. Therefore it is natural to prove the uniqueness theorem under restrictions imposed on the stress vector. Such a problem posed in the book Knops and Payne [1] was solved by T. Buchukuri (see Buchukuri [1]).

In Buchukuri and Gegelia [1-4] Theorem 2 is proved by the method of asymptotic representation of solutions of the external problems in a neighbourhood of infinity. The same theorem is proved in Kondratyev and Olejnik $[1,2]$ by a different method based on the Korn's inequality. The method of asymptotic representation of solutions turned out suitable also for other models of the elasticity theory; in particular, for models described by systems of equations containing both the higher derivatives and the derivatives of first and zero orders (equations of couple-stress elasticity and equations of a microporous medium).

2.3. Uniqueness Theorems for Oscillation Problems. If a homogeneous isotropic elastic medium is subjected to the action of external forces periodic in time, then it is natural to assume that displacement, strain and stress components depend on time in the same manner. Such a state of an elastic medium is called stationary elastic oscillation. Equations of this state are written in the form $A\left(\partial_{x}\right) u+\omega^{2} u=0$, where $\omega$ is the oscillation frequency, $A\left(\partial_{x}\right)$ is the differential operator of classical elasticity determined 
by the formula (2). The density of the medium in question is assumed to be equal to unity without loss of generality.

V. Kupradze proved (see Kupradze (1)) the following theorem.

Theorem 3. Any external basic problem of stationary elastic oscillation cannot have two regular solutions $u$ satisfying the conditions

$$
\begin{gathered}
\lim _{|x| \rightarrow \infty} u^{(p)}(x)=0, \quad \lim _{|x| \rightarrow \infty} u^{(s)}(x)=0 \\
\lim _{|x| \rightarrow \infty} r\left(\frac{\partial u^{(p)}(x)}{\partial r}-i k_{1} u^{(p)}(x)\right)=0 \\
\lim _{|x| \rightarrow \infty} r\left(\frac{\partial u^{(s)}(x)}{\partial r}-i k_{2} u^{(s)}(x)\right)=0
\end{gathered}
$$

where

$$
\begin{aligned}
& r=|x|, \quad k_{1}^{2}=\omega^{2}(\lambda+2 \mu)^{-1}, \quad k_{2}^{2}=\omega^{2} \mu^{-1}, \quad i^{2}=-1, \\
& u^{(p)}=\frac{1}{k_{2}^{2}-k_{1}^{2}}\left(\Delta+k_{2}^{2}\right) u, \quad u^{(s)}=-\frac{1}{k_{2}^{2}-k_{1}^{2}}\left(\Delta+k_{1}^{2}\right) u .
\end{aligned}
$$

By analogy with the radiation conditions of Sommerfeld (A. Sommerfeld, V. Kupradze, F. Rellich), the conditions (9), (10) are called the conditions of elastic radiation (Kupradze (1)).

Theorem 3 is valid for an isotropic medium. Its extension to an anisotropic medium turned out a difficult problem which was nevertheless solved.

Let $A\left(\partial_{x}\right)$ be the matrix differential operator of the classical elasticity theory of anisotropic media (see (6)). We shall consider equations of stationary oscillation

$$
A\left(\partial_{x}\right) u(x)+\omega^{2} u(x)=0
$$

It is assumed that

1) $\nabla_{\xi} \phi(\xi, \omega) \neq 0$ for $\phi(\xi, \omega)=0, \xi \in \mathbb{R}^{3}$;

2) the total curvature of the manifold $\phi(\xi, \omega)=0$ vanishes nowhere.

Here $\phi(\xi, \omega) \equiv \operatorname{det}\left(I \omega^{2}-A(\xi)\right), \xi \in \mathbb{R}^{3}, I \equiv\left\|\delta_{k j}\right\|_{3 \times 3}$.

With these assumptions the equation $\phi(\xi, \omega)=0$ determines three compact, convex, two-dimensional surfaces $S_{1}, S_{2}, S_{3}$ which do not intersect. Moreover, for any point $x \in \mathbb{R}^{3} \backslash\{0\}$ there exists on $S_{j}$ a unique point $\xi^{j}$ such that $n\left(\xi^{j}\right)$ is directed along the vector $x$. By $n\left(\xi^{j}\right)$ we denote the external normal to the surface $S_{j}$ at the point $\xi^{j}(j=1,2,3)$.

Let $W_{m}\left(\Omega^{-}\right)$denote a set of vectors $v=\left(v_{1}, v_{2}, v_{3}\right) \in C^{1}\left(\Omega^{-}\right)$satisfying in a neighbourhood of infinity the conditions

$$
v_{k}(x)=\sum_{j=1}^{3} v_{k}^{j}(x), \quad v_{k}^{j}(x)=O\left(|x|^{-1}\right),
$$




$$
\begin{gathered}
\lim _{r \rightarrow \infty} r\left(\frac{\partial v_{k}^{j}(x)}{\partial r}+i(-1)^{m}\left(\frac{x}{r} \xi^{j}\right) v_{k}^{j}(x)\right)=0, \\
j=1,2,3 ; \quad|x|=r ; \quad m=1 \text { or } m=2 .
\end{gathered}
$$

D. Natroshvili proved the following theorem:

Theorem 4. Any external basic problem of stationary elastic oscillation of anisotropic media cannot have two regular solutions of the class $W_{m}\left(\Omega^{-}\right)$.

To prove the theorem D. Natroshvili had constructed a fundamental matrix $\Gamma(x, \omega, m)$ of the operator $A\left(\partial_{x}\right)+I \omega^{2}$. This matrix belongs to the class $W_{m}\left(\mathbb{R}^{3} \backslash\{0\}\right)$. It is constructed by means of the limiting absorption principle from the fundamental matrix $\Gamma\left(x, \tau_{\varepsilon}\right)$ of the operator $A\left(\partial_{x}\right)-\tau_{\varepsilon}^{2} I$ $\left(\tau_{\varepsilon}=\varepsilon+i \omega\right)$, which vanishes at infinity more rapidly than any negative power of $|x|$ (cf. Vainberg [1]).

2.4. Asymptotic Representation of Solutions at Infinity. The asymptotic representation of solutions in a neighbourhood of infinity discussed in Subsection 2.2 is based on the Green and Somigliana formulas which, in turn, are constructed by means of the fundamental solution.

Let us consider a system of equations

$$
A_{i k}\left(\partial_{x}\right) u_{k}=0 \quad\left(A\left(\partial_{x}\right) u=0\right),
$$

where $A_{i k}\left(\partial_{x}\right)$ is the differential operator determined by the formula

$$
A_{i k}\left(\partial_{x}\right)=a_{i j k l} \frac{\partial^{2}}{\partial x_{j} \partial x_{l}}
$$

$u=\left(u_{1}, \ldots, u_{n}\right)$ is the unknown vector, $x=\left(x_{1}, \ldots, x_{m}\right)$ is a point from $\mathbb{R}^{m}, a_{i j k l}$ are the constants satisfying the conditions $a_{i j k l}=a_{i l k j}$. In addition, we require of the system (13) to be elliptic. This is equivalent to the condition

$$
\forall \xi=\left(\xi_{1}, \ldots, \xi_{m}\right) \in \mathbb{R}^{m} \backslash\{0\}: \operatorname{det} A(\xi)=\operatorname{det}\left\|A_{i k}(\xi)\right\|_{n \times n} \neq 0 .
$$

If it is assumed that $m=n=3$ and $a_{i j k l}=a_{k l i j}=a_{j i k l}$, then the system (13) turns into the system of the classical elasticity theory for an anisotropic medium.

Let us consider the conjugate system of equations

$$
A_{i k}^{*}\left(\partial_{x}\right) v_{k}=0 \quad\left(A^{*}\left(\partial_{x}\right) v=0\right),
$$

where

$$
A_{i k}^{*}\left(\partial_{x}\right)=a_{k l i j} \frac{\partial^{2}}{\partial x_{j} \partial x_{l}}=a_{k j i l} \frac{\partial^{2}}{\partial x_{j} \partial x_{l}}=A_{k i}\left(\partial_{x}\right) .
$$


In John [1] there is constructed a fundamental matrix $\phi=\left\|\phi_{k s}\right\|_{n \times n}$ such that

1) $\phi_{k s} \in C^{\infty}\left(\mathbb{R}^{m} \backslash\{0\}\right), \forall x \in \mathbb{R}^{m} \backslash\{0\}: A_{i k}\left(\partial_{x}\right) \phi_{k s}(x)=0$;

2) $\forall t \neq 0, \forall x \in \mathbb{R}^{m} \backslash\{0\}: \partial^{\alpha} \phi(t x)=t^{-|\alpha|-m+2} \partial^{\alpha} \phi(x)$, where $\alpha=$ $\left(\alpha_{1}, \ldots, \alpha_{m}\right)$ is an arbitrary multiindex;

3) $\forall x \in \mathbb{R}^{m}$ :

$$
\lim _{\delta \rightarrow 0} \int_{\partial B(x, \delta)} T_{i k}^{*}\left(\partial_{y}, \nu\right) \phi_{k s}(y-x) d_{y} S=\delta_{i s},
$$

where $B(x, \delta)$ is the ball with center at the point $x$ and radius $\delta$, and

$$
\begin{gathered}
T^{*}\left(\partial_{y}, \nu\right)=\left\|T_{i k}^{*}\left(\partial_{y}, \nu\right)\right\|_{n \times n} \\
T_{i k}^{*}\left(\partial_{y}, \nu\right)=a_{k j i l} \nu_{j} \frac{\partial}{\partial y_{l}}=T_{k i}\left(\partial_{y}, \nu\right), \quad T\left(\partial_{y}, \nu\right)=\left\|T_{k i}\left(\partial_{y}, \nu\right)\right\|_{n \times n} .
\end{gathered}
$$

The following theorem is valid (see Buchukuri and Gegelia [1-4]):

Theorem 5. Let $\Omega$ be a domain from $\mathbb{R}^{m}$ containing a neighbourhood of infinity, $u$ be a solution of the system (13) in the domain $\Omega$, belonging to the class $C^{2}(\Omega)$ and satisfying one of the conditions below:

$$
\begin{aligned}
& \lim _{r \rightarrow \infty} \frac{1}{r^{m+p+1}} \int_{B(0, r) \backslash B(0, r / 4)}|u(z)| d z=0, \\
& \lim _{|z| \rightarrow \infty} \frac{|u(z)|}{|z|^{p+1}}=0, \quad \int_{\Omega} \frac{|u(z)| d z}{1+|z|^{m+p+1}}<+\infty,
\end{aligned}
$$

where $p$ is a nonnegative integer. Then in a neighbourhood of infinity the following asymptotic representation of $u=\left(u_{1}, \ldots, u_{n}\right)$ holds:

$$
u_{s}(x)=\sum_{|\alpha| \leq p} c_{s}^{(\alpha)} x^{\alpha}+\sum_{|\beta| \leq q} d_{k}^{(\beta)} D^{\beta} \phi_{k s}(x)+\psi_{s}(x),
$$

where $c_{s}^{(\alpha)}=$ const, $d_{s}^{(\beta)}=$ const, $\alpha=\left(\alpha_{1}, \ldots, \alpha_{m}\right)$ and $\beta=\left(\beta_{1}, \ldots, \beta_{m}\right)$ are multiindices, $q$ is an arbitrary nonnegative integer, and

$$
\left|D^{\gamma} \psi_{s}(x)\right| \leq \frac{c}{|x|^{m+|\gamma|+q+1}}
$$

$c=$ const, $\gamma=\left(\gamma_{1}, \ldots, \gamma_{m}\right)$ is an arbitrary multiindex.

It should be emphasized that each of the three terms in the right-hand side of the representation (21) is a solution of the system (13).

Theorem 5 implies the following corollaries: 
Corollary 1. If $u \in C^{2}(\Omega), \forall x \in \Omega: A\left(\partial_{x}\right) u(x)=0$ and $u(x)=o(1)$ $(m>2), u(x)=o(\ln |x|)(m=2)$ as $|x| \rightarrow \infty$, then there exists the limit $\lim _{|x| \rightarrow \infty} u(x)=\left(c_{1}, \ldots, c_{n}\right)$.

Corollary 2. If $u \in C^{2}(\Omega), \forall x \in \Omega: A\left(\partial_{x}\right) u(x)=0$ and $u(x)=o(1)$ $(m \geq 2)$ as $|x| \rightarrow \infty$, then for any multiindex $\alpha$ :

$$
D^{\alpha} u(x)=O\left(|x|^{2-m-|\alpha|}\right) \quad(m>2), \quad D^{\alpha} u(x)=O\left(|x|^{1-|\alpha|}\right) \quad(m=2) .
$$

In particular,

$$
\begin{array}{ccc}
u(x)=O\left(|x|^{2-m}\right), & T\left(\partial_{x}, \nu\right) u(x)=O\left(|x|^{1-m}\right) \quad(m>2), \\
u(x)=O\left(|x|^{-1}\right), & T\left(\partial_{x}, \nu\right) u(x)=O\left(|x|^{-2}\right) \quad(m=2) .
\end{array}
$$

2.5. Solutions of Boundary Value Problems with Power Growth at Infinity. Theorem 5 makes it possible to investigate boundary value problems in more general formulations than the classical ones.

Let $\Omega^{+}$be a bounded domain from $\mathbb{R}^{m}$ with the smooth boundary $\partial \Omega^{+} \equiv$ $S$. Let $\Omega^{-} \equiv \mathbb{R}^{m} \backslash\left(\Omega^{+} \cup S\right)$.

Problem $(I)_{c s}^{-}$. In the domain $\Omega^{-}$find a vector $u=\left(u_{1}, \ldots, u_{n}\right)$ of the class $C^{2}\left(\Omega^{-}\right) \cap C^{1}\left(\bar{\Omega}^{-}\right)$, satisfying the conditions

$$
\begin{aligned}
\forall x \in \Omega^{-}: & A\left(\partial_{x}\right) u(x)=0, \quad \forall y \in S:(u(y))^{-}=\varphi(y), \\
& u(x)=o\left(|x|^{p+1}\right) \quad \text { as } \quad|x| \rightarrow \infty .
\end{aligned}
$$

Here $A\left(\partial_{x}\right)$ is the differential operator determined by the formula (13), $\varphi$ is a given function $\left(\varphi=\left(\varphi_{1}, \ldots, \varphi_{n}\right)\right)$ on $S$, and $p$ is a nonnegative integer.

Let us denote by $G_{c s}^{I}(p, m)$ the set of all solutions of the corresponding homogeneous $(\varphi=0)$ problem.

T. Buchukuri proved (see Buchukuri and Gegelia [3]) the following

Theorem 6. $G_{c s}^{I}(p, m)$ is a finite-dimensional linear set whose dimension is calculated by the formula $\operatorname{dim} G_{c s}^{I}(p, m)=n\left(C_{p+m-1}^{m-1}+C_{p+m-2}^{m-1}\right)$; here $C_{r}^{s}$ is the binomial coefficient; $C_{r}^{s}=0$ if $s>r$.

Corollary 1. If $\varphi \in H^{\alpha}\left(\partial \Omega^{-}\right)(\alpha>0)$, then the problem $(I)_{c s}^{-}$is solvable and the solution is represented in the form $u=u^{(0)}+u^{(p)}$, where $u^{(0)}$ is a solution of the problem $(I)_{c s}^{-}$, vanishing at infinity, and $u^{(p)}$ is an arbitrary element of the set $G_{c s}^{I}(p, m)$.

Similar theorems and corollaries hold for all the basic problems, also for the main contact problem. However, it is difficult to calculate dimension of the set of solutions of the homogeneous problems which in the classical formulations have nontrivial solutions. 
Corollary 2. In the classical theory of elasticity, $m=n=3$ and

$$
\operatorname{dim} G_{c s}^{I}(p, 3)=3\left(C_{p+2}^{2}+C_{p+1}^{2}\right) .
$$

Therefore we shall have three linear independent solutions of the first basic problem, satisfying the condition $\lim _{|x| \rightarrow \infty} \frac{u(x)}{|x|}=0$.

Note that the investigation of problems of the type $(I)_{c s}^{-}$is far from completion. Dimensions of spaces of the type $G_{c s}^{I}(p, m)$ have not been calculated for other problems of elasticity. Nothing has been done in this direction in couple-stress elasticity and thermoelasticity, as well as for other models.

2.6. Asymptotic Representation in the Couple-Stress Theory of Elasticity. To prove the validity of a representation of the form (21) for solutions of a system of the couple-stress theory of elasticity turned out to be a difficult task. A system of the basic equations of this theory for an anisotropic medium is written in the form

$$
\begin{gathered}
c_{i j l k} \frac{\partial^{2} u_{k}}{\partial x_{j} \partial x_{l}}-c_{j i l m} \varepsilon_{k l m} \frac{\partial \omega_{k}}{\partial x_{j}}=0, \\
c_{j m l k} \varepsilon_{i j m} \frac{\partial u_{k}}{\partial x_{l}}+c_{j i l k}^{\prime} \frac{\partial^{2} \omega_{k}}{\partial x_{j} \partial x_{l}}-c_{j m l p} \varepsilon_{i j m} \varepsilon_{k l p} \omega_{k}=0,
\end{gathered}
$$

$u=\left(u_{1}, u_{2}, u_{3}\right)$ is a displacement vector, $\omega=\left(\omega_{1}, \omega_{2}, \omega_{3}\right)$ is a rotation vector, $\varepsilon_{i j k}$ is the Levy-Civita symbol, $c_{i j l k}=$ const, $c_{i j l k}^{\prime}=$ const.

The system (24) contains both the second order derivatives of the unknown vectors and the first and zero order derivatives. The latter circumstance essentially complicates the character of the fundamental matrix of the system (24). This matrix does not possess the property 2) from Subsection 2.4. Yet, T. Buchukuri managed to obtain the estimates of the fundamental matrix needed to prove the validity of a representation of the form (21) (see Buchukuri and Gegelia [4]).

An asymptotic representation of the form (21) has not been obtained for many models of the elasticity theory in the case of an anisotropic medium.

2.7. Mixed Basic Problem of the Elasticity Theory. Mixed basic problems of the elasticity theory - when a boundary condition of one type, say, displacement is given on one part of the boundary and a condition of another type, say, stress is given on the remaining part of the boundary are reduced to SIE on open surfaces. Mixed plane problems are reduced to SIE on open contours.

The SIE theory on open contours is completely elaborated both in the classes of smooth functions and in the classes of summable functions (Muskhelishvili [2], Muskhelishvili and Kveselava [1], N. Vekua [1] and others). These results and their development enabled G. Mandzhavidze, V. 
Kupradze and T. Burchuladze to bring to the end the investigation of mixed plane problems of elasticity.

The SIE theory on open surfaces in the classes of Hölder functions has not been developed to a sufficient extent; some results in this direction are obtained by R. Kapanadze in Kapanadze [2]. For the time being mixed problems of the elasticity theory have not been investigated with the required completeness (see Subsection 2.12).

2.8. Properties of Solutions of the Basic Equations of Elasticity near Singular Points. As said previously, the fundamental solution of the considered system plays a special role in potential methods. This solution satisfies the system everywhere except the origin at which it has a singularity. Such a solution is a displacement field produced by the force source concentrated at the origin. Singular solutions are generated by other force sources as well. For example, the so-called double force produces a field of a higher singularity than the fundamental solution. It is natural to try to find all singular solutions of the system under consideration, or, speaking more exactly, all solutions of the system which, at given points, possess a concentrated singularity of any order, say, of the power order. The following theorem provides the answer to this problem (see Buchukuri and Gegelia $[1-4])$.

Theorem 7. Let $\Omega$ be a domain from $\mathbb{R}^{m}, y \in \Omega, u=\left(u_{1}, \ldots, u_{n}\right)$ be $a$ solution of the system (13) in the domain $\Omega \backslash\{y\}$ and $\forall x \in \Omega \backslash\{y\}$ :

$$
|u(x)| \leq \frac{c}{|x-y|^{\gamma}}
$$

where $c=$ const, $\gamma \geq 0$. Then $\forall x \in \Omega \backslash\{y\}$ :

$$
u(x)=u^{0}(x)+\sum_{|\alpha| \leq[\gamma]+2-m}\left(\partial_{x}^{\alpha} \phi(x-y)\right) a^{(\alpha)},
$$

where $u^{0}$ is a regular solution of the system (13) in the domain $\Omega(u \in$ $\left.C^{2}(\Omega)\right), \alpha=\left(\alpha_{1}, \ldots, \alpha_{m}\right)$ is a multiindex, $[\gamma]$ is the integer part of the number $\gamma, a^{(\alpha)}=\left(a_{1}^{(\alpha)}, \ldots, a_{m}^{(\alpha)}\right), a_{i}^{(\alpha)}=$ const, $\phi$ is the fundamental matrix of the system (13).

It should be noted that the second term in $(26)$ is absent when $[\gamma]+2-$ $m<0$. Moreover, replacing (25) by the condition

$$
u(x)=o\left(\frac{1}{|x-y|^{q}}\right),
$$

where $q$ is a natural number, we can perform summation in the representation (26) up to $q+1-m$. 
Theorem 7 precisely establishes the properties of solutions of the system (13) in the neighbourhood of an isolated singular point. The representation (26) immediately implies the theorem on a removable singularity.

Corollary 1. Let $\Omega$ be a domain in $\mathbb{R}^{m}, u=\left(u_{1}, \ldots, u_{n}\right)$ belong to the class $C^{2}(\Omega \backslash\{y\}), y \in \Omega$ and $\forall y \in \Omega \backslash\{y\}: A\left(\partial_{x}\right) u(x)=0$. Let, besides,

$$
u(x)=o\left(\frac{1}{|x-y|^{m-2}}\right), \quad m>2 ; \quad u(x)=o(\ln |x-y|), \quad m=2 .
$$

Then $y$ is a removable singularity for $u$, i.e., there exists a limit $\lim _{x \rightarrow y} u(x)$ $\equiv u(y)$ and if we complete the definition of $u$ at the point $y$ by the value $u(y)$, then $u \in C^{2}(\Omega)$.

The representation (26) also implies yet another theorem frequently used in applications.

Corollary 2. Let the conditions of Theorem 7 be fulfilled and $\gamma>m-2$ $(m \geq 2)$ in the estimate (25). Then for any multiindex $\alpha$

$$
\left|D^{\alpha} u(x)\right| \leq \frac{c}{|x-y|^{[\gamma]+|\alpha|}} .
$$

In particular,

$$
\left|T\left(\partial_{x}, \nu\right) u(x)\right| \leq \frac{c}{|x-y|[\gamma]+1},
$$

where $T$ is the stress operator.

Theorem 7 can be used to investigate the basic problems for the system (13) in more general formulations than their classical counterparts.

Let $\Omega$ be a bounded domain from $\mathbb{R}^{m}$ with the smooth boundary $S \equiv \partial \Omega$ and $y^{(1)}, \ldots, y^{(r)}$ be a set of ponts lying in this domain.

Problem $(I)_{c s}$. Find a vector $u=\left(u_{1}, \ldots, u_{m}\right)$ of the class $C^{2}\left(\Omega \backslash\left\{y^{(1)}, \ldots, y^{(r)}\right\}\right) \cap C^{1}\left(S \cup \Omega \backslash\left\{y^{(1)}, \ldots, y^{(r)}\right\}\right)$, satisfying the conditions

$$
\begin{gathered}
\forall x \in \Omega \backslash\left\{y^{(1)}, \ldots, y^{(r)}\right\}: A\left(\partial_{x}\right) u(x)=F(x), \\
\forall y \in S: \lim _{\Omega \ni x \rightarrow y \in S} u(x)=\varphi(y), \\
\forall x \in \Omega \backslash\left\{y^{(1)}, \ldots, y^{(r)}\right\}:|u(x)| \leq \sum_{i=1}^{r} \frac{c}{\left|x-y^{(i)}\right|^{p_{i}}} .
\end{gathered}
$$

Here $F$ and $\varphi$ are given vector-functions, $c=$ const, and $p_{i}$ are given nonnegative numbers.

This problem will also be referred to as problem $(30)_{F},(31)_{\varphi},(32)$. 
Theorem 8. The homogeneous problem $(I)_{c s}$, i.e., the problem $(30)_{0}$, $(31)_{0}$, (32) has exactly

$$
n \sum_{i=1}^{r}\left(C_{p_{i}+1}^{m-1}+C_{p_{i}}^{m-1}\right)
$$

linearly independent solutions.

If $\varphi \in H^{\alpha}(S)(\alpha>0)$, the nonhomogeneous problem $(I)_{c s}$, or more exactly the problem $(30)_{0},(31)_{\varphi},(32)$ has a solution $u$ which is represented in the form $u=u^{(\varphi)}+u^{(0)}$, where $u^{(\varphi)}$ is a solution of the problem $(30)_{0}$, $(31)_{\varphi}$, regular in the domain $\Omega$, and $u^{(0)}$ is an arbitrary element of the set $G\left((I)_{c s}\right)$. Here $G\left((I)_{c s}\right)$ denotes the set of all solutions of the homogeneous problem $(30)_{0},(31)_{0},(32)$.

The investigation of the second basic problem demands some effort to overcome certain difficulties. For the sake of simplicity let us consider a system of the classical elasticity theory $m=n=3$.

Problem $(I I)_{c s}$. Let $\Omega$ be a bounded domain from $\mathbb{R}^{3}$, containing the origin. It is required to find a vector $u=\left(u_{1}, u_{2}, u_{3}\right)$ in the domain $\Omega_{1}=$ $\Omega \backslash\{0\}$ by the conditions $u \in C^{2}\left(\Omega_{1}\right) \cap C^{1}\left(S \cup \Omega_{1}\right)$,

$$
\begin{gathered}
\forall x \in \Omega_{1}: A\left(\partial_{x}\right) u(x)=0, \\
\forall y \in S: \lim _{\Omega \ni x \rightarrow y \in S} T\left(\partial_{x}, \nu\right) u(x)=0, \\
\forall x \in \Omega_{1}:|u(x)| \leq \frac{c}{|x|^{p}} .
\end{gathered}
$$

Let $u$ be a solution of the problem (34)-(36). Then, by virtue of Theorem 7 , it is represented in the form

$$
u_{k}(x)=u_{k}^{(0)}(x)+\sum_{|\alpha| \leq p-1} c_{\alpha j} D^{\alpha} \Gamma_{k j}(x),
$$

where $u^{(0)}$ is a regular solution of (13) in the domain $\Omega$.

Here $\Gamma$ is the matrix of fundamental solutions of the classical elasticity theory.

Taking into account (35) and the easily verifiable equalities

$$
\begin{gathered}
\int_{S} T_{i k}\left(\partial_{y}, \nu(y)\right) u_{k}(y) d_{y} S=0 \\
\int_{S} \varepsilon_{i j k} y_{j} T_{k l}\left(\partial_{y}, \nu(y)\right) u_{l}(y) d_{y} S=0
\end{gathered}
$$


we find from (37) that

$$
\begin{gathered}
u_{k}=u_{k}^{(0)}+c_{11} \frac{\partial \Gamma_{k 1}}{\partial x_{1}}+c_{22} \frac{\partial \Gamma_{k 2}}{\partial x_{2}}+c_{33} \frac{\partial \Gamma_{k 3}}{\partial x_{3}}+c_{12}\left(\frac{\partial \Gamma_{k 2}}{\partial x_{1}}-\frac{\partial \Gamma_{k 1}}{\partial x_{2}}\right)+ \\
+c_{13}\left(\frac{\partial \Gamma_{k 3}}{\partial x_{1}}-\frac{\partial \Gamma_{k 1}}{\partial x_{3}}\right)+c_{23}\left(\frac{\partial \Gamma_{k 3}}{\partial x_{2}}-\frac{\partial \Gamma_{k 2}}{\partial x_{3}}\right)+\sum_{2 \leq \alpha \leq p-1} c_{\alpha j} D^{\alpha} \Gamma_{k j} .
\end{gathered}
$$

Thus any solution of the problem (34)-(36) can be represented as the sum of a solution $u^{(0)}$ regular in $\Omega$ and a linear combination of vectors $\psi^{(r)}=\left(\psi_{1}^{(r)}, \psi_{2}^{(r)}, \psi_{3}^{(r)}\right)$ with

$$
\begin{gathered}
\psi_{k}^{(1)}=\frac{\partial \Gamma_{k 1}}{\partial x_{1}}, \quad \psi_{k}^{(2)}=\frac{\partial \Gamma_{k 2}}{\partial x_{2}}, \quad \psi_{k}^{(3)}=\frac{\partial \Gamma_{k 3}}{\partial x_{3}}, \quad \psi_{k}^{(4)}=\frac{\partial \Gamma_{k 2}}{\partial x_{1}}-\frac{\partial \Gamma_{k 1}}{\partial x_{2}}, \\
\psi_{k}^{(5)}=\frac{\partial \Gamma_{k 3}}{\partial x_{1}}-\frac{\partial \Gamma_{k 1}}{\partial x_{3}}, \quad \psi_{k}^{(6)}=\frac{\partial \Gamma_{k 3}}{\partial x_{2}}-\frac{\partial \Gamma_{k 2}}{\partial x_{3}}
\end{gathered}
$$

and $\left(D^{\alpha} \Gamma_{1 j}, D^{\alpha} \Gamma_{2 j}, D^{\alpha} \Gamma_{3 j}\right)_{2 \leq \alpha \leq p-1}(j=1,2,3)$.

The above reasoning leads to

Theorem 9. $\operatorname{dim} G\left((I I)_{c s}\right)=n_{p}+6$, where $n_{p}=0$ for $p \leq 1$ and $n_{p}=3 p^{2}-6$ for $p \geq 2$.

This theorem belongs to T. Buchukuri (see Buchukuri and Gegelia [3]).

As one may conclude from this survey, the investigation of problems with concentrated singularities has not been completed even in the classical elasticity theory. They have not been studied at all in thermoelasticity, couple-stress elasticity, elasticity with independent dilatation and so on.

We would like to note that solutions of problems with concentrated singularities contain arbitrary constants. These constants can be used to construct solutions possessing some additional properties, for example, a property to minimize a functional or a property to take given values at given points.

2.9. Dynamic Problems. The investigation of dynamic problems or, as they are frequently called, initial-boundary problems in the elasticity theory is fraught with some difficulties. In these problems it is required to define a dynamic state of the medium, i.e., it is required to find in the cylinder $\mathrm{C} \equiv \Omega \times[0, \infty]$ a solution of the system

$$
A\left(\partial_{x}\right) u(x, t)-\rho^{2} \frac{\partial^{2} u(x, t)}{\partial t^{2}}=\rho F(x, t),
$$

which satisfies the initial condition

$$
\lim _{t \rightarrow 0} u(x, t)=\varphi(x), \quad \lim _{t \rightarrow 0} \frac{\partial u(x, t)}{\partial t}=\psi(x)
$$


at each point $x$ in the domain $\Omega$ and one of the boundary conditions of the basic problems.

Dynamic problems were initially investigated by Hilbert space methods (G. Fichera, O. Maisaia and others) and afterwards by potential methods (V. Kupradze, T. Burchuladze, L. Magnaradze, T. Gegelia, R. Rukhadze, R. Kapanadze, R. Chichinadze and others).

Using the Laplace transform V. Kupradze and T. Burchuladze reduced the dynamic problems to the boundary value problems for an elliptic system $A\left(\partial_{x}\right) u(x, \tau)-\tau^{2} v(x, \tau)=F(x, \tau)$. The complex parameter $\tau$ that also participates in the boundary conditions is the result of the formal Laplace transformation with respect to the time variable.

Thus the initial boundary problems are formally reduced to the elliptic boundary value problems with a complex parameter.

Such a reduction of the dynamic problem has long been known in mathematical physics. The investigation begins after this procedure, as it is necessary to substantiate the inverse Laplace transformation by the parameter $\tau$. For such a procedure V. Kupradze and T. Burchuladze used the Green tensors. Presently, there are several approaches to obtain estimates of the Green tensors. One of them is the representation of the Green tensors in the form of a composition of singular kernels (T. Gegelia, D. Natroshvili, R. Kapanadze, R. Chichinadze).

The methods of solution of dynamic problems proposed by V. Kupradze and T. Burchuladze were afterwards extended to other models. Especially intensive investigations are being carried out in this direction in the thermoelasticity theory and its modern models of Green-Lindsay and LordShulman (see Burchuladze and Gegelia [1]).

2.10. Contact (Interface) Problems of the Elasticity Theory. The potential methods turned out efficient also in investigating contact and boundary-contact problems. Let $\Omega$ and $\Omega_{k}(k=1, \ldots, n)$ be domains with the connected smooth boundaries $\partial \Omega$ and $\partial \Omega_{k}$. Note that $\bar{\Omega}_{i} \cap \bar{\Omega}_{j}=\varnothing$ if $i \neq j$ and $\bar{\Omega}_{i} \subset \Omega$. We introduce the notation:

$$
\Omega_{0} \equiv \Omega \backslash \bigcup_{k=1}^{n} \Omega_{k}, \quad S \equiv \partial \Omega \bigcup_{k=r+1}^{n} \partial \Omega_{k}(r<n), \quad L \equiv \bigcup_{k=1}^{r} \partial \Omega_{k} .
$$

Let the domain $\Omega_{0}$ be filled up by an elastic medium with the Lamé constants $\lambda_{0}$ and $\mu_{0}$, and the domains $\Omega_{k}(k=1, \ldots, r)$ by elastic media with the Lamé constants $\lambda_{k}$ and $\mu_{k}$. Thus a nonhomogeneous elastic medium with piecewise-homogeneous structure occupies the domain $D=\cup_{k=0}^{r} \Omega_{k}$ and $\Omega_{i}(i=r+1, \ldots, n)$ are hollow inclusions.

The case is admitted when $\Omega$ is the entire space $\mathbb{R}^{3}$; then $\partial \Omega=\varnothing$. We also may encounter the case $r=n$.

The basic boundary-contact problem consists in finding in the domain $\Omega_{k}(k=0, \ldots, r)$ a regular solution of the equation $A^{(k)}\left(\partial_{x}\right) u=\rho_{k} F$, 
which satisfies one of the boundary conditions of the basic problems on the boundary $S$, and the contact conditions on the contact (interface) surfaces $\partial \Omega_{k}(k=1, \ldots, r)$ : displacement and boundary stress jumps are given $\left(A^{(k)}\left(\partial_{x}\right)\right.$ is defined by (2) where $\lambda$ and $\mu$ are replaced by $\lambda_{k}$ and $\left.\mu_{k}\right)$.

We may also consider a more general problem when different boundary conditions are given on the surfaces $\partial \Omega, \partial \Omega_{r+1}, \ldots, \partial \Omega_{n}$ - this is a mixed boundary-contact problem.

When the dynamic state is considered, to the above conditions we must add initial conditions.

V. Kupradze was the first to investigate the boundary-contact problem by the potential method. He proved that this problem is solvable when the Poisson coefficients of the contacting media coincide. Subsequently, these problems were investigated without any restrictions on the Poisson coefficients in Basheleishvili, Gegelia [1] and, for problems of thermoelasticity, in Jentsch $[1,3,14]$.

L. Jentsch [5-8] and afterwards V. Kupradze introduced into consideration other contact problems. In these problems instead of displacement and stress jumps we are given jumps of normal components of the displacement and the stress vectors and values of the tangent components of the stress vector (problem G) or jumps of normal components of the displacement and stress vectors and values of the tangent components of the displacement vector (problem H). Other contact conditions are also possible. Various type of contact problems of elasticity and thermoelasticity were investigated by V. Kupradze, L. Jentsch, R. Katamadze, R. Gachechiladze, O. Maisaia and others and for the anisotropic case in Jentsch and Natroshvili [1].

We would like to note that the true contact problems which occasionally are also called Picone problems were investigated even earlier by the Hilbert space methods (J. Lions, S. Campanato, G. Fichera). G. Fichera and afterwards O. Maisaia, R. Gachechiladze and M. Kvinikadze studied contact problems for isotropic as well as for anisotropic and homogeneous media.

More complicated contact problems were investigated when, for example, the assumptions of classical elasticity are valid for media occupying the domain $\Omega_{i}(i=1, \ldots, \nu ; \nu<r)$, and the assumptions of couple-stress elasticity are valid for other media occupying $\Omega_{i}(i=\nu+1, \ldots, r)$. Problems of this kind are treated in the papers of O. Maisaia and M. Kvinikadze. Some new properties of the solutions have been found.

So far it has been assumed that $\bar{\Omega}_{i} \subset \Omega$, but if $\Omega_{i} \subset \Omega$, then $\partial \Omega_{i} \cap \partial \Omega \neq \varnothing$ or $\Omega_{i} \cap \Omega=\varnothing$, but $\partial \Omega_{i} \cap \partial \Omega \neq \varnothing$. In such situations the contact problem becomes essentially more complicated. Using pseudodifferential operators, O. Chkadua has obtained the first results in this direction. In the plane case similar problems of bimodal type were investigated with the aid of the theory of singular integral equations with fixed singularities (see Duduchava [1]) and Mellin techniques by L. Jentsch [10-25]. 
2.11. New Models of Thermoelasticity. In recent years intensive investigations have involved new various models of the elasticity theory which take into account interactions of different mechanical and nonmechanical fields. Thermoelasticity is the natural generalization of the classical elasticity theory. The classical model of the elasticity theory does not take into account temperature changes. But deformation is always accompanied by temperature changes and a temperature change is always accompanied by deformation even in the absence of external force. The physical fundamentals of the thermoelasticity theory were developed by J.M.K. Duhamel, W. Voigt, H. Jeffreys, M.A. Biot and discussed by G. Cattaneo, I. Müller, S. Kaliski, W. Nowacki, A.E. Green, K.A. Lindsay, H.W. Lord, Y. Shulman, J. Ignaczak, Ya. Podstrigach, Yu. Kolyano, and others.

Equations of the classical thermoelasticity theory are written in the form

$$
\begin{gathered}
A\left(\partial_{x}\right) v(x, t)-\gamma \operatorname{grad} \theta(x, t)-\rho \frac{\partial^{2} v(x, t)}{\partial t^{2}}=F(x, t), \\
\Delta \theta(x, t)-\frac{1}{\varkappa} \frac{\partial \theta(x, t)}{\partial t}+\eta \frac{\partial}{\partial t} \operatorname{div} v(x, t)=F_{4}(x, t),
\end{gathered}
$$

where $A\left(\partial_{x}\right)$ is the matrix differential Lamé operator (see Kupradze (1)), $v=\left(v_{1}, v_{2}, v_{3}\right)$ is the displacement vector, $\theta$ is a temperature change, $F=$ $\left(F_{1}, F_{2}, F_{3}\right)$ and $F_{4}$ are given by external force and $\gamma, \varkappa, \rho, \eta$ are physical constants.

V. Kupradze and his pupils T. Burchuladze and N. Kakhniashvili were the first to apply potential methods to thermoelasticity. They developed completely the theory of boundary value, initial-boundary and contact problems, studied the steady state oscilation problems and investigated other aspects of the theory.

The classical model of thermoelasticity does not take into account the heat flow time, which led to the well-known paradoxes in this theory. Hence new improved models were created, of which the models of Green-Lindsay and Lord-Shulman enjoy particular popularity. The Green-Lindsay model is described by the system

$$
\begin{aligned}
& A\left(\partial_{x}\right) v(x, t)-\gamma \operatorname{grad} \theta(x, t)-\gamma \tau_{1} \frac{\partial}{\partial t} \operatorname{grad} \theta(x, t)-\rho \frac{\partial^{2} v(x, t)}{\partial t^{2}}=F(x, t), \\
& \Delta \theta(x, t)-\frac{1}{\varkappa} \frac{\partial \theta(x, t)}{\partial t}+\frac{\tau_{t}}{\varkappa} \frac{\partial^{2} \theta(x, t)}{\partial t^{2}}+\eta \frac{\partial}{\partial t} \operatorname{div} v(x, t)=F_{4}(x, t),
\end{aligned}
$$

and the Lord-Shulman model by the system

$$
\begin{gathered}
A\left(\partial_{x}\right) v(x, t)-\gamma \operatorname{grad} \theta(x, t)-\rho \frac{\partial^{2} v(x, t)}{\partial t^{2}}=F(x, t), \\
\Delta \theta(x, t)-\frac{1}{\varkappa} \frac{\partial \theta(x, t)}{\partial t}+\frac{\tau_{0}}{\varkappa} \frac{\partial^{2} \theta(x, t)}{\partial t^{2}}+\eta \frac{\partial}{\partial t} \operatorname{div} v(x, t)+
\end{gathered}
$$




$$
+\eta \tau_{t} \frac{\partial^{2}}{\partial t^{2}} \operatorname{div} v(x, t)=F_{4}(x, t) .
$$

For these models and also for models in which diffusion and couple stresses are taken into account T. Burchuladze and his pupils constructed fundamental solutions, derived Green formulas and representations of solutions, constructed the corresponding potentials, established radiation conditions, obtained estimates of Green tensors and investigated both boundary value and initial-boundary problems (see Burchuladze and Gegelia [1]).

Mention should further be made of the approximate method of Fourier series which in the foreign literature is called the Riesz-Fisher-Kupradze method. T. Burchuladze showed that this method is efficient also for just mentioned models (see Burchuladze and Gegelia [1]). Methods of constructing explicit solutions for some domains bounded by a system of planes also work well (see Burchuladze [1]).

2.12. Application of Pseudodifferential Operators. This subsection contains an outline of the investigation of the mixed problems of elasticity by the potential method using pseudodifferential operators (see Prössdorf [2], Maz'ya [1], Eskin [1], Boutet de Monvel [1], Triebel [1, 2], Shamir [1], Duduchava [3], Shargorodsky [1, 2], Duduchava, Natroshvili and Shargorodsky [1], Natroshvili, Chkadua and Shargorodsky [1], Natroshvili and Shargorodsky [1] and others).

Let $\Omega^{+}$be a bounded domain from $\mathbb{R}^{3}$ with a smooth boundary $S$ of the class $C^{k}(k \geq 4) ; \Omega^{-} \equiv \mathbb{R}^{3} \backslash\left(\Omega^{+} \cup S\right)$. Let $S$ be represented as $S=\bar{S}_{1} \cup \bar{S}_{2}$, where $S_{1} \cap S_{2}=\varnothing, \bar{S}_{1} \cap \bar{S}_{2} \equiv L$. It is assumed that $S_{1}$ and $S_{2}$ and also $L$ are smooth manifolds. $S_{1}$ and $S_{2}$ are two-dimensional surfaces with boundary and $L$ is a closed curve (without an edge).

Problem $\left[\Omega^{+}, S_{1}, S_{2}\right]$. Find a solution of the system (13) in the domain $\Omega^{+}$, satisfying the conditions

$$
\left.u\right|_{S_{1}} ^{+}=\varphi,\left.\quad T\left(\partial_{z}, n\right) u\right|_{S_{2}} ^{+}=\psi .
$$

The mixed problem for the domain $\Omega^{-}$is formulated similarly, but in that case, to preserve the uniqueness theorem, the solution must satisfy the condition $u(x)=o(1)$ for $|x| \rightarrow \infty$.

Note that the formulation of the boundary value problems $\left[\Omega^{+}, S_{1}, S_{2}\right]$ and $\left[\Omega^{-}, S_{1}, S_{2}\right]$ is not rigorous because we have not indicated those functional classes where solutions are to be found. This refinement will be made later.

Let $\phi$ be the fundamental solution of the system (13). Consider the single-layer potential

$$
V(g)(x) \equiv \int_{S} \phi(x-y) g(y) d_{y} S,
$$


and denote its restriction on $S$ by $V_{S}(g) . V_{S}(g): S \rightarrow \mathbb{R}^{3}$ is a pseudodifferential operator (PDO) of order -1 .

If $u$ is the solution of the problem $\left[\Omega^{+}, S_{1}, S_{2}\right]$, then it satisfies the system (13) in the domain $\Omega^{+}$and the boundary condition

$$
\left.u\right|_{S} ^{+}=f,
$$

where $f: S \rightarrow \mathbb{R}^{3}$ and coincides with $\varphi$ on $S_{1}$. The values of $f$ on $S_{2}$ are unknown. This function can be written in the form $f=\phi_{0}+\varphi_{0}$, where $\phi_{0}$ is some known function coinciding with $\varphi$ on $S_{1}$, and $\varphi_{0}$ is the desired function on $S$, which is equal to zero on $S_{1}$. Thus, if we find $\varphi_{0}$, then for defining $u$ we obtain the first basic problem (13), (49).

The solution of the problem (13), (49) will be sought for in the form of the simple-layer potential $u=V(g)$. Then from (49) it follows that $V_{S}(g)=f=\phi_{0}+\varphi_{0}$. It can be proved that the operator $V_{S}(g)$ is invertible in the corresponding pair of functional spaces. That is why $g=V_{S}^{-1}\left(\phi_{0}+\varphi_{0}\right)$ and therefore

$$
u=V\left(V_{S}^{-1}\left(\phi_{0}+\varphi_{0}\right)\right)=V\left(V_{S}^{-1}\left(\phi_{0}\right)\right)+V\left(V_{S}^{-1}\left(\varphi_{0}\right)\right) .
$$

The solution $u$ represented by the formula (50) satisfies the system (13) and the first boundary condition from (47). We must choose $\varphi_{0}$ such that the second boundary condition from (47) be fulfilled. Calculating $\left.T\left(\partial_{x}\right) u\right|_{S} ^{+}$, we obtain

$$
\begin{gathered}
\left.T\left(\partial_{z}, n\right) u\right|_{S} ^{+}(z)=-\frac{V_{S}^{-1}\left(\phi_{0}\right)(z)}{2}+\int_{S} T \partial_{z} \phi(z-y) V_{S}^{-1}\left(\phi_{0}\right)(y) d_{y} S- \\
-\frac{V_{S}^{-1}\left(\varphi_{0}\right)(z)}{2}+\int_{S} T \partial_{z} \phi(z-y) V_{S}^{-1}\left(\varphi_{0}\right)(y) d_{y} S .
\end{gathered}
$$

For $z \in S_{2}$ this equality implies

$$
\begin{gathered}
-\frac{V_{S}^{-1}\left(\varphi_{0}\right)(z)}{2}+\int_{S} T \partial_{z} \phi(z-y) V_{S}^{-1}\left(\varphi_{0}\right)(y) d_{y} S= \\
=\psi(z)+\frac{V_{S}^{-1}\left(\phi_{0}\right)(z)}{2}-\int_{S} T \partial_{z} \phi(z-y) V_{S}^{-1}\left(\phi_{0}\right)(y) d_{y} S,
\end{gathered}
$$

which is a pseudodifferential equation on the manifold $S_{2}$ with the boundary $L$.

Note that the PDO contained in (51) does not possess the transmission property (see Eskin [1], Boutet de Monvel [1], Rempel and Schulze [1]) and the theory of such equations in Hölder spaces $C^{m+\alpha}$ has not as yet been developed. That is why we have to investigate the equation (51) in Bessel 
potential spaces $H_{p}^{s}$ and in Besov spaces $B_{p, q}^{s}$ (see Burchuladze and Gegelia [1], Triebel [1, 2]). The PDO theory for these spaces is worked out in Shamir [1], Duduchava [3], Shargorodsky [1, 2]. After the uniqueness and existence theorems are proved in these spaces, the Hölder continuity of the solution is established by means of the embedding theorem.

Before we proceed to formulate the results, two circumstances have to be noted: for some particular cases we have expilicit formulas (see, e.g., Vorovich, Aleksandrov and Babeshko [1]) for the solution of the problem $\left[\Omega^{+}, S_{1}, S_{2}\right]$, according to which at points $x$ near the edge $L$ the solution behaves like $\sqrt{\rho(x)}$, where $\rho(x)$ is the distance from the point $x$ to $L$. Therefore, generally speaking, the solution does not belong to the class $W_{p}^{2}$ for $p \geq 4 / 3$, to the class $W_{p}^{1}$ for $p \geq 4$ and to the class $C^{\alpha}$ for $\alpha>1 / 2$.

Besides, if the solution of the problem $\left[\Omega^{+}, S_{1}, S_{2}\right]$ is sought for in the Sobolev spaces $W_{p}^{2}\left(\Omega^{+}\right)$or $W_{p}^{1}\left(\Omega^{+}\right)$, then the equation (13) can be understood in the sense of generalized functions. Then the respective sense should be given to the boundary conditions (47), too, understanding by them the trace of the corresponding functions. However, in the case of the space $W_{p}^{1}\left(\Omega^{+}\right)$there arises a complication because the derivative of the function from the class $W_{p}^{1}\left(\Omega^{+}\right)$belongs to the class $L_{p}\left(\Omega^{+}\right)$and its trace on $S$ is not determined. Therefore we should give sense to the second boundary condition of (47) by means of Green's formula and generated by it duality. This can be done thanks to the fact that the solution of the problem is not an arbitrary function from the class $W_{p}^{1}\left(\Omega^{+}\right)$, but a function satisfying the equation (13) in $\Omega^{+}$.

We finally obtain the validity of the following theorem (see Duduchava, Natroshvili and Shargorodsky [1], Natroshvili, Chkadua and Shargorodsky [1]).

Theorem 10. Let $4 / 3<p<4(1<p<4 / 3)$ and $\varphi \in B_{p, p}^{1-1 / p}\left(S_{1}\right)$, $\psi \in B_{p, p}^{-1 / p}\left(S_{2}\right)\left(\varphi \in B_{p, p}^{2-1 / p}\left(S_{1}\right), \psi \in B_{p, p}^{1-1 / p}\left(S_{2}\right)\right)$. Then the problem $\left[\Omega^{+}, S_{1}, S_{2}\right]$ has the unique solution of the class $W_{p}^{1}\left(\Omega^{+}\right)\left(W_{p}^{2}\left(\Omega^{+}\right)\right)$. If $\varphi \in B_{t, t}^{s}\left(S_{1}\right)$ and $\psi \in B_{t, t}^{s-1}\left(S_{2}\right)$, then the solution $u$ of the class $W_{p}^{1}\left(\Omega^{+}\right)$ also belongs to the class $H_{t}^{s+1 / t}\left(\Omega^{+}\right)$. If $\varphi \in B_{t, q}^{s}\left(S_{1}\right), \psi \in B_{t, q}^{s-1}\left(S_{2}\right)$, then $u \in B_{t, q}^{s+1 / t}\left(\Omega^{+}\right)$. If $\varphi \in C^{\alpha}\left(\bar{S}_{1}\right), \psi \in B_{\infty, \infty}^{\alpha-1}\left(S_{2}\right)\left(0<\alpha \leq \frac{1}{2}\right)$, then $u \in C^{\alpha^{\prime}}\left(\Omega^{+}\right)$with $\alpha^{\prime}<\alpha$.

Here $1<t<\infty, 1 \leq q \leq \infty, 1 / t-1 / 2<s<1 / t+1 / 2$.

A similar theorem holds for the problem $\left[\Omega^{-}, S_{1}, S_{2}\right]$, too.

The method described can be used to investigate mixed problems for the oscillation equation (11) and the pseudooscillation equation $A\left(\partial_{x}\right) u-\tau^{2} u=$ $0, \tau=\sigma+i \omega, \sigma \neq 0$.

The initial-boundary mixed problems for the dynamic state are treated by the conventional technique, i.e., by reducing them using the Laplace trans- 
form to the mixed problems of pseudooscillation (see Natroshvili, Chkadua and Shargorodsky [1]).

Problems of the mathematical theory of cracks evoke special interest. They are also successfully investigated by the method of pseudodifferential equations (see Duduchava, Natroshvili and Shargorodsky [1] and Natroshvili, Chkadua and Shargorodsky [1]).

2.13. Optimization and Control Problems in the Elasticity Theory. Let $\Omega$ be a domain from $\mathbb{R}^{3}$ with a sufficiently smooth boundary $S$. Consider some basic problem of the elasticity theory:

Find in the domain $\Omega$ a regular solution of the equation

$$
A\left(\partial_{x}\right) u(x)=F(x),
$$

by the boundary condition

$$
\forall y \in S:\left(D\left(\partial_{y}\right) u\right)(y)=f(y),
$$

where $F=\left(F_{1}, F_{2}, F_{3}\right) f=\left(f_{1}, f_{2}, f_{3}\right)$ are given vectors in $\bar{\Omega}$ and on $S$, respectively. $A\left(\partial_{x}\right)$ is a differential operator of the elasticity theory (see (2) or $(6)), D\left(\partial_{y}\right)$ is the boundary operator of some basic problem (if the first problem is considered, then $D\left(\partial_{y}\right)$ is the identity operator; if the second problem is considered, then $D\left(\partial_{y}\right)$ is the boundary stress operator and so on).

Under certain additional restrictions (see Kupradze (1)), which we shall assume to be fulfilled, the problem (52), (53) has the unique solution $u$. Let us consider some functional $l(u)$ of the solution $u$. It is obvious that $u$ depends on the parameters of the problem (52), (53), i.e., on the coefficients of the operator $A\left(\partial_{x}\right)$, on the right-hand side $F$ of the equation (52) and on the boundary data $f$. These parameters can be used to control the functional $l(u)$. We may, for example, pose a question of finding in the defined functional space $H$ a vector $F$ minimizing the functional $l$.

If a similar problem is considered for elastic stationary oscillation (in that case the equation (52) is replaced by the equation (11)), then to the considered parameters of the problem we should add an oscillation frequency and then the control of the functional can be effected by any parameter (or parameters) of the problem.

Similar problems can be investigated for the dynamic state, too. For example, the following problem has been investigated:

$$
\begin{gathered}
\forall(x, t) \in Q: A\left(\partial_{x}\right) u(x, t)-\rho(x) \frac{\partial^{2} u(x, t)}{\partial t^{2}}=0, \\
\forall x \in \bar{\Omega}: u(x, 0)=0, \quad \frac{\partial u(x, 0)}{\partial t}=0 ; \quad \forall(x, t) \in \Sigma: u(x, t)=g(x, t),
\end{gathered}
$$


where $\Omega$ is a domain from $\mathbb{R}^{3}, Q=\Omega \times(0, T), \Sigma=\partial \Omega \times(0, T), g$ is a given vector on $\Sigma$.

If $g$ is a sufficiently smooth vector and $\partial \Omega$ is a sufficiently smooth surface, then the problem (54) has a sufficiently smooth solution $u$.

Let $\varphi$ and $\psi$ be vectors of the class $L^{2}(\Omega)$ given on $\Omega$. Consider the functional

$$
J(g) \equiv \int_{\Omega}\left(|u(x, t)-\varphi(x)|^{2}+\left|\frac{\partial u(x, t)}{\partial t}-\psi(x)\right|^{2}\right) d x .
$$

Theorem 11. There exists a number $T_{0}$ such that if $T \geq T_{0}$, then $\inf J(g)=0, g \in C^{\infty}(\Omega)$.

Some investigations involve problems of the control of various functionals by solutions of problems of thermoelasticity, by solutions of singular integral equations and so on.

Problems of control have not been as yet considered with sufficient completeness in the elasticity theory. Only the first results have been obtained (see works by O. Maisaia, A. Jorbenadze, T. Tsutsunava).

2.14. Noncorrect and Nonclassical Problems. Various nonclassical (see Vorovich, Aleksandrov, Babeshko [1], Maz'ya [2] and others) and noncorrect problems of the elasticity theory have been investigated. In these problems the sets with given boundary data or contacting media are not bounded or have additional boundary conditions on one part of the boundary and free boundary conditions on the remaining part.

We shall mention one noncorrect problem which was investigated by the quasi-inversion method (O. Maisaia)

Let $G_{1}, G_{2}, G_{3}$ be bounded domains from $\mathbb{R}^{3}$ and $\bar{G}_{1} \subset G_{2}, \bar{G}_{2} \subset G_{3}$. Let $\Omega_{1} \equiv G_{2} \backslash \bar{G}_{1}$ and $\Omega_{2} \equiv G_{3} \backslash \bar{G}_{2}$. Then $\partial \Omega_{1}=\partial G_{2} \cup \partial G_{1}$ and $\partial \Omega_{2}=$ $\partial G_{3} \cup \partial G_{2}$. Functions $u^{(1)}$ and $u^{(2)}$ are to be found, for which

$$
\begin{aligned}
\forall x \in \Omega_{k}: & A^{(k)}\left(\partial_{x}\right) u^{(k)}(x)=0, \quad k=1,2 ; \\
\forall y \in \partial G_{1}: & u^{(1)}(y)=\varphi(y), \quad T^{(1)}\left(\partial_{y}, n\right) u^{(1)}(y)=\psi(y) ; \\
\forall z \in \partial G_{2}: & u^{(1)}(z)-u^{(2)}(z)=0, \\
& T^{(1)}\left(\partial_{z}, n\right) u^{(1)}(z)-T^{(2)}\left(\partial_{z}, n\right) u^{(2)}(z)=0 .
\end{aligned}
$$


2.15. Potential Methods in the Plane Elasticity Theory. Potential methods are used to solve and to investigate many plane problems of elasticity, in problems of anisotropic plate bending, in boundary-contact problems and so on. These problems are reduced to equivalent integral equations, which makes it possible to represent the solutions of problems by means of potentials whose kernels are written in terms of elementary functions. These potentials are applied to obtain solutions in series or in quadratures for some particular cases (a half-plane, a strip, an ellipse and so on). For example, an effective solution is obtained for the mixed problem for the whole plane with an elliptic cavity or with cuts arranged on the straight line and so on (Jentsch [20, 21, 24], M. Basheleishvili, Sh. Zazashvili, Zh. Rukhadze and others). The concept of equivalent potentials of single-layer type was applied by J. Maul (see Jentch, Maul [1], Maul [1,2] to very general mixed contact problems.

If an elastic medium occupies a plane domain with a piece-wise smooth boundary, then we obtain singular integral equations containing singular integrals with fixed singularities. Such equations and their applications are treated in Duduchava [1].

Mention should also be made of the investigations conducted by complex potentials (see works by G. Mandzhavidze, E. Obolashvili, R. Bantsuri, G. Janashia and others).

2.16. Solutions in Quadratures of Boundary Value Problems of the Elasticity Theory for a Ball and the Whole Space with a Spherical Cavity. Methods of constructing effetive solutions of problems of this theory play a special role in the theory of continuum mechanics. By effectiveness we understand the construction of solutions either in elementary functions or in series or in quadratures. To avoid misunderstanding we shall always indicate clearly in what form the solutions are constructed.

Sufficiently detailed information on effective solutions of the spatial problems of elasticity and thermoelasticity can be found in Kupradze (1). We shall dwell here on some most noteworthy results obtained in this direction by the potential methods.

Numerous works starting from the the well-known memoirs of Lord Kelvin to the present-day studies are devoted to solution of the basic problems for a ball and the entire space with a spherical cavity. It is not our intention here to give a full account of the history of this question. We wish only to note that in 1972 D. Natroshvili succeeded in summing series of spherical functions that give solutions of the basic problems of the elasticity theory and in representing the obtained solutions in the form of quadratures (D. Natroshvili). After Professor G. Fichera learnt about D. Natroshvili's results, he sent the Tbilisi collegues the paper of R. Marcolongo where the solutions in quadratures of the basic problems were obtained by a differ- 
ent method without resorting to series as far back as 1904. The method and results of Marcolongo became the subjectmatter of many interesting investigations. We shall discourse on some of them below.

To grasp the essence of Marcolongo's method which is in turn based on V. Cerruti's ideas, let us consider how this method is applied to the solution of the problems of classical elasticity.

Let $B^{+}$be a ball with centre at the origin and radius $\rho, B^{-} \equiv \mathbb{R}^{3} \backslash \overline{B^{+}}$, $S \equiv \partial B^{+}=\partial B^{-}$.

The basic problems of the elasticity theory are formulated as follows: Find in $\overline{B^{+}}$a continuous vector $u$, which in $B^{+}$is a solution of the system (2), by the boundary conditions: on the boundary $S$ we are given displacement $f$ (Problem $(I)^{+}$) or stress $f$ (Problem $(I I)^{+}$), or tangential stress components $g$ and normal displacement component $l$ (Problem $\left.(I I I)^{+}\right)$, or tangential displacement components $g$ and a normal stress component $l$ (Problem $\left.(I V)^{+}\right)$, or a linear combination of displacements and stresses (Problem $(V)^{+}$). The problems for the unbounded domain $B^{-}$are formulated in the same manner.

The following theorems are proved (see Gegelia and Chichinadze [2]):

Theorem 12. If

$$
u(x)=v(x)+\frac{\rho^{2}-r^{2}}{2} \operatorname{grad} \psi(x),
$$

where the vector-function $v$ and the scalar function $\psi$ are continuous in $B^{+}$ or $B^{-}$and satisfy the conditions

$$
\begin{gathered}
\Delta v=0, \quad \Delta \psi=0, \quad\left(D_{r}+\alpha\right) \psi=\beta \operatorname{div} v, \\
\alpha=\frac{\mu}{\lambda+3 \mu}, \quad \beta=\frac{\lambda+\mu}{\lambda+3 \mu}, \quad r=|x|, \quad D_{r}=r \frac{d}{d r}=\sum x_{i} \frac{\partial}{\partial x_{i}},
\end{gathered}
$$

then the vector $u$ is a solution of the system (2) in $B^{+}$or $B^{-}$. And conversely, if $u$ is a continuous solution of the system (2) in $B^{+}$or $B^{-}$, then there exist a vector $v$ and a scalar $\psi$ continuous in $B^{+}$or $B^{-}$for which the conditions (59) and (60) are fulfilled.

The solution of Problem $(I)^{+}$is sought for in the form (59). Then to define $v$ we obtain the Dirichlet problem $\forall x \in B^{+}: \Delta v(x)=0, \forall y \in S$ : $v^{+}(y)=f(y)$, whose solution is given by the Poisson formula

$$
v(x)=\Pi(f)(x) \equiv \frac{1}{4 \pi \rho} \int_{S} \frac{\rho^{2}-|x|^{2}}{|y-x|^{3}} f(y) d_{y} S .
$$

To define $\psi$ we obtain an ordinary differential equation $r \frac{d \psi}{d r}+\alpha \psi=$ $\beta \operatorname{div} \Pi(f)$. 
Finally, the solution of Problem $(I)^{+}$is given in the form

$$
u(x)=\int_{S} \mathcal{K}(x, y) f(y) d_{y} S,
$$

where $\mathcal{K}=\left\|\mathcal{K}_{i j}\right\|_{3 \times 3}$,

$$
\mathcal{K}_{i j}(x, y)=\frac{1}{4 \pi \rho}\left(\frac{\rho^{2}-|x|^{2}}{|y-x|^{3}} \delta_{i j}+\frac{\beta\left(\rho^{2}-|x|^{2}\right)}{2} \frac{\partial^{2}}{\partial x_{i} \partial x_{j}} \int_{0}^{1}\left(\frac{\rho^{2}-|\tau x|^{2}}{|y-\tau x|^{3}}-\frac{1}{\rho}\right) \frac{d \tau}{\tau^{2-\alpha}}\right) .
$$

The representation (62) implies

Theorem 13. If $f \in C(S)$, then the solution of Problem $(I)^{+}$is given in quadratures in the form of the integral (62) and is the unique classical solution (from the class $C^{2}\left(B^{+}\right) \cap C\left(\overline{B^{+}}\right)$) of this problem.

The solution of Problem $(I)^{-}$is also given in quadratures and it is also the unique solution in the class of functions satisfying the condition at infinity $u(x)=o(1)$.

Similar theorems are valid for Problems $(I I)^{ \pm}$and $(V)^{ \pm}$, while for Problems $(I I I)^{ \pm}$and $(I V)^{ \pm}$more rigid restrictions are imposed on the boundary data $g$ and $l$ (see Chichinadze [4], Gegelia, Chichinadze [2]).

Note that it is not convenient to represent the solution in the form (59) for Problems $(I I I)^{ \pm}$and $(I V)^{ \pm}$. For these problems we must modify the representation (59) and Theorem 12.

2.17. Solution in Quadratures of Boundary Value Problems of the Thermoelasticity Theory. Marcolongo's method is applied with some modifications in the thermoelasticity theory as well. In classical thermoelasticity a static state is described by a separated system of equations

$$
\mu \Delta u(x)+(\lambda+\mu) \operatorname{grad} \operatorname{div} u(x)-\gamma \operatorname{grad} \theta(x)=0, \quad \Delta \theta(x)=0 .
$$

If we find $\theta$ from the Laplace equation and substitute it in the first equation (62), then we shall obtain a system of nonhomogeneous equations of the classical elasticity theory. This simple way of investigating the stationary state is quite suitable for proving theorems of the existence and uniqueness of solutions of boundary value problems, but cannot be used for constructing effective solutions. Formulas for representation of solutions of nonhomogeneous equations are rather inefficient and not suitable for our purposes.

Boundary value problems for the system (63) can be solved in quadratures directly, applying a theorem similar to Theorem 12. A lot of problems of the form $(p, q)^{ \pm}(p=1,2,3,4,5, q=1,2)$, where $p$ corresponds to the problem $(p)^{ \pm}$of the elasticity theory and $q$ to the problem $(q)^{ \pm}$of harmonic 
functions, have been posed in the thermoelasticity theory. All these problems are solved in quadratures and theorems of the type of Theorem 13 (see Gegelia and Chichinadze [2]) are proved.

Problems for a sphere have not been solved for nonclassical models of thermoelasticity such as, for example, the Lord-Shulman or Green-Lindsay theory (see Burchuladze and Gegelia [1]).

2.18. Problems for the Polyharmonic Equation. The method of representation of solutions by means of harmonic functions proved to be suitable in solving problems for a higher order equation. Consider the polyharmonic equation

$$
\Delta^{\nu+1} u(x)=0
$$

where $\Delta^{\nu+1}=\Delta\left(\Delta^{\nu}\right), \Delta^{1}=\Delta$ is the Laplace operator and $\nu$ is a positive integer.

In regard to the equation (64) it is of interest to investigate the Lauricella, Riquier and mixed problems (M. Nicolesco, I. Vekua, K. Miranda). In these problems it is required to find a continuous solution of the equation (64) in the domain by the following boundary equations:

Lauricella problem: $\forall y \in S:\left(\frac{d^{k} u}{d n^{k}}\right)^{+}(y)=f_{k}(y), k=0, \ldots, \nu$;

Riquier problem: $\forall y \in S:\left(\Delta^{k} u\right)^{+}(y)=f_{k}(y), k=0, \ldots, \nu$;

Mixed problem: $\forall y \in S$ :

$$
\begin{gathered}
\left(\frac{d^{k} u}{d n^{k}}\right)^{+}(y)=f_{k}(y), \quad k=0, \ldots, \mu, \quad 1 \leq \mu<\nu, \\
\left(\Delta^{k}\right)^{+}(y)=f_{k}(y), \quad k=\mu+1, \ldots, \nu .
\end{gathered}
$$

All these problems are solved in quadratures (see Chichinadze $[5,6]$, Gegelia and Chichinadze [2]).

2.19. Problems for Elastic Mixtures. In recent years researchers have displayed great interest in the investigation of elastic mixtures. We shall not discuss here whether the respective models are viable or not. For information concerning this question we refer the reader to Khoroshun and Soltanov [1], Natroshvili, Jagmaidze and Svanadze [1], Truesdell and Toupin [1], Green and Naghdi [1], Steel [1], Green and Steel [1], Atkin, Chadwik and Steel [1], Tiersten and Jahanmir [1], Villaggio [1].

Thorough consideration has been given to the two-component mixture whose equations are written in the form

$$
\begin{aligned}
& a_{1} \Delta \stackrel{(1)}{u}+b_{1} \operatorname{grad} \operatorname{div} \stackrel{(1)}{u}+c \Delta \stackrel{(2)}{u}+d \operatorname{grad} \operatorname{div} \stackrel{(2)}{u}=F_{1}, \\
& c \Delta \stackrel{(1)}{u}+d \operatorname{grad} \operatorname{div} \stackrel{(1)}{u}+a_{2} \Delta \stackrel{(2)}{u}+b_{2} \operatorname{grad} \operatorname{div} \stackrel{(2)}{u}=F_{2},
\end{aligned}
$$


where $a_{1}, a_{2}, b_{1}, b_{2}, c, d$ are the elastic constants, $\stackrel{(1)}{u}=\left(\stackrel{(1)}{u}_{1}, \stackrel{(1)}{u}_{2}, \stackrel{(1)}{u}_{3}\right)$ and $\stackrel{(2)}{u}=\left(\stackrel{(2)}{u}_{1}, \stackrel{(2)}{u}_{2}, \stackrel{(2)}{u}_{3}\right)$ are the displacement vectors.

The boundary value problems are posed and comletely investigated for the system (65) in Natroshvili, Jagmaidze and Svanadze [1] by means of potential methods. Problems of thermoelastic mixtures have also been investigated. All these problems are solved in quadratures for the ball and the whole space with a spherical cavity in Chichinadze [5], Gegelia and Chichinadze [2].

2.20. Boundary Value Problems of Macropolar Fluid Flow. If a fluid contains a countless quantity of solid particles in the form of an admixture, the flow of such a fluid will not obey satisfactorily the classical Navier-Stokes model. Eringen [1] gives an example confirming this phenomenon. In such situations it is better to represent the flow both as the displacement of the point and as its rotation about itself. This is the moment theory of flow or, speaking differently, the flow with regard to the fluid microstructure. The model of such a flow was created by A. Eringen, also by D. Kondif and I. Dagler. It however turned out to be rather complicated, since it involved a nonlinear system of partial equations containing seven equations with respect to seven unknowns

$$
\begin{gathered}
(\mu+\alpha) \Delta v(x, t)+2 \alpha \operatorname{rot} \omega(x, t)-\operatorname{grad} p(x, t)+\rho f(x, t)= \\
=\frac{\partial v(x, t)}{\partial t}+v_{k}(x, t) \frac{\partial v(x, t)}{\partial x_{k}}, \\
(\nu+\beta) \Delta \omega(x, t)+(\varepsilon+\nu-\beta) \operatorname{grad} \operatorname{div} \omega(x, t)+2 \alpha \operatorname{rot} v(x, t)- \\
-4 \alpha \omega(x, t)+\rho \mathcal{G}(x, t)=I \frac{\partial \omega(x, t)}{\partial t}+J v_{k}(x, t) \frac{\partial \omega(x, t)}{\partial x_{k}}, \\
\operatorname{div} v(x, t)=0 .
\end{gathered}
$$

This is a closed system of nonstationary flow of a viscous noncompressible homogeneous isotropic micropolar fluid, $v=\left(v_{1}, v_{2}, v_{3}\right)$ is the flow velocity, $\omega=\left(\omega_{1}, \omega_{2}, \omega_{3}\right)$ is a rotation, $p$ is the pressure, and $\alpha, \beta, \mu, \nu, \tau, \rho$ are the physical constants.

Like in the classical Navier-Stokes model, two linearization variants of the system (66) are considered, namely systems obtained by analogy with the Stokes linearization and with the Ozeen linearization.

All the basic problems formulated for the obtained linearization systems are investigated in Chichinadze [1], Buchukuri and Chichinadze [1, 2].

In addition to the above-mentioned references, various questions of the micropolar fluid flow are treated in work by N. Ramkinson. 
2.21. Effective Solutions of Boundary Value Problems of Fluid Flow. It should be noted that the method of representing solutions of the Stokes-linearized classical Navier-Stokes model by means of harmonic functions proved to be a convenient tool in solving the fluid problem for a sphere.

The above-mentioned homogeneous system of equations is written in the form

$$
\mu \Delta v(x)-\operatorname{grad} p(x)=0, \quad \operatorname{div} v(x)=0,
$$

where $v=\left(v_{1}, v_{2}, v_{3}\right)$ is a velocity vector, $p$ is a pressure, $\mu$ is the viscosity coefficient.

For this case the following representation theorem is valid.

Theorem 14. If

$$
\begin{gathered}
v(x)=u(x)+x\left(2 \mathcal{D}_{r}+1\right) \psi(x)-r^{2} \operatorname{grad} \psi(x)+\frac{\rho^{2}-r^{2}}{2} \operatorname{grad} \psi(x), \\
p(x)=-\mu\left(2 \mathcal{D}_{r}+1\right) \psi(x), \\
\Delta u=0, \quad \Delta \psi=0, \quad 2 \mathcal{D}_{r}^{2} \psi+4 \mathcal{D}_{r} \psi+3 \psi=-\operatorname{div} u,
\end{gathered}
$$

then the pair $(v, p)$ gives the solution of the system $(68)$ in $B^{+}$and $B^{-}$.

The converse statement is valid, too. Here $B^{+} \equiv\left\{x \in \mathbb{R}^{3}|| x \mid<\rho\right\}$, $B^{-} \equiv \mathbb{R}^{3} \backslash \overline{B^{+}}, S \equiv \partial \Omega^{+}=\partial \Omega^{-}, \mathcal{D}_{r}=r \frac{d}{d r}, r \equiv|x|$.

This theorem is used to prove

Theorem 15. If $f \in C(S)$ and the necessary condition of solvability

$$
\int_{S} y f(y) d_{y} S=0,
$$

is fulfilled, then the pair $(v, p)$ defined by the equalities

$$
\begin{gathered}
v(x)=\frac{1}{4 \pi \rho} \int_{S} \frac{\rho^{2}-|x|^{2}}{|x-y|^{3}} f(y) d_{y} S+ \\
+\frac{\rho^{2}-x^{2}}{4 \pi \rho} \operatorname{grad} \operatorname{div} \int_{S}\left(\frac{1}{|x-y|}+\frac{3 \chi(x, y)}{2 \rho^{2}}\right) f(y) d_{y} S \\
p(x)=-\frac{\mu}{2 \pi \rho} \operatorname{div} \int_{S}\left(\frac{\rho^{2}-|x|^{2}}{|x-y|^{3}}+\frac{1}{|x-y|}-\frac{3 \chi(x, y)}{2 \rho^{2}}\right) f(y) d_{y} S+p_{0}
\end{gathered}
$$

gives all classical solutions of the first basic problem.

Here $p_{0}$ is an arbitrary constant and $\chi(x, y)=|x-y|+\frac{x \cdot y}{\rho} \ln ((|x-y|+$ $\left.\rho)^{2}-|x|^{2}\right)$. 
Similar theorems are valid also for the other problems of fluid flow.

\section{REFERENCES}

M.A. Assaf and L. Jentsch 1. On the elasticity theory of microporous solids. Z. angewandte Math. Mech. 72(1992), No. 8, 321-340.

R.J. Atkin, P. Chadwick and T.R. Steel 1. Uniqueness in the linear theory of a mixture of two elastic solids. Internat. J. Engrg. Sci. 7(1969), No. 6, 571-577.

M.O. Basheleishvili and T.G. Gegelia 1. On the basic spatial boundary value problems for composite isotropic elastic media. (Russian) Dokl. Akad. Nauk SSSR 160(1965), No. 1, 50-53.

2. In vestigation of the third and fourth boundary value problems of statics for an isotropic elastic body. (Russian) Proc. I. Vekua Inst. Appl. Math. (Tbiliss. Gos. Univ. Inst. Prikl. Mat. Trudy) 3(1972), 29-67.

M.O. Basheleishvili and Sh.P. Zazashvili 1. A mixed boundary value problem of the plane theory of statics of an anisotropic elastic body. (Russian) Collect. Sci. works Georgian Politech. Inst. (Mezhvuzovski sbornik nauchn. trudov Gruzin. Politekh. Inst.), Tbilisi, 1985, 53-62.

2. Effective solution of the mixed boundary value problem for an infinite anisotropic plane with an elliptic hole. (Russian) Proc. I. Vekua Inst. Appl. Math. (Tbiliss. Gos. Univ. Inst. Prikl. Mat. Trudy) 46(1992), 24-30.

S.M. Belonosov and K.A. Chernous 1. Boundary value problems for the Navier-Stokes equation. (Russian) Nauka, Moscow, 1985.

L. Boutet de Monvel 1. Boundary problems for pseudo-differential operators. Acta Math. 126(1971), 11-51.

J. Boussinesq 1. Applications des potentiales à l'étude de l'équilibre et du mouvements des solides élastiques. Paris, 1885.

T.V. Buchukuri 1. On the solutions of external static boundary value problems of elastisity with stress tensor at infinity. (Russian) Bull. Acad. Sci. Georgia 142(1991), No. 1, 33-36.

2. Computation of Poisson type integrals. Proc. Georgian Acad. Sci. Math. 1(1993), No. 6, 643-655.

T.V. Buchukuri and T.G. Gegelia 1. Behaviour of stresses and displacements near a singular point. (Russian) Sem. I. Vekua Inst. Appl. Math. Tbilisi St. Univ. Reports (Sem. Inst. Prikl. Mat. Dokl.) 2(1986), No. 2, 25-28.

2. On the uniqueness of solutions of the basic problems of the elasticity theory for infinite domains. (Russian) Differentsial'nye Uravneniya 25(1989), No. 9, 1556-1565.

3. Qualitative properties of solutions of the basic equations of the elasticity theory near singular points. (Russian) Trudy Tbiliss. Mat. Inst. Razmadze 90(1988), 40-67. 
4. On the uniqueness theorems for the external problems of the couplestress theory of elasticity. Proc. Georgian Acad. Sci. Math. 1(1993), No. $2,143-157$.

T.V. Buchukuri and R.K. Chichinadze 1. Boundary value problems of stationary flow of a noncompressible viscous micropolar fluid in the case of Ozeens linearization. (Russian) Trudy Tbiliss. Mat. Inst. Razmadze 86(1991), 29-60.

2. Two-dimensional problems of stationary flow of a noncompressible viscous fluid in the case of Ozeen's linearization. Proc. Georian Acad. Sci. Math. 1(1993). No. 3, 281-297.

T.V. Burchuladze 1. Nonstationary problems of generalized elastothermodiffusion for inhomogeneous media. Proc. Georgian Acad. Sci. Math. 1(1993), No. 6, 657-669.

T.V. Burchuladze and T.G. Gegelia 1. Development of the potential method in the theory of elasticity. (Russian) Metsniereba, Tbilisi, 1985, $1-226$.

R.K. Chichinadze 1. Boundary value problems of stationary flow of a noncompressible viscous flow. (Russian) Trudy Tbiliss. Mat. Inst. Razmadze 75(1984), 111-132.

2. Solution of the first boundary value problem for the Stokes equation in the case of a domain with a spherical boundary. (Russian) Bull. Acad. Sci. Georgian SSR 124(1986), No. 2, 277-280.

3. Boundary value problems for the Stokes equation in the case of a domain with a spherical boundary. (Russian) Trudy Tbiliss. Mat. Inst. Razmadze 87(1987), 136-149.

4. On the boundary value problems of the elasticity theory for the ball. (Russian) Trudy Tbiliss. Mat. Inst. Razmadze 96(1991), 97-122.

5. Solution of the first boundary value problem of the theory of elastic mixtures. (Russian) Bull. Acad. Sci. Georgia 141(1991), No. 2, 293-296.

6. Solution of the Lauricella boundary value problem for the ball. (Russian) Bull. Acad. Sci. Georgia 146(1992), No. 1, 135-140.

7. The Riquier boundary value problem for the ball. (Russian) Bull. Acad. Sci. Georgia 146(1992), No. 3, 515-520.

S.L. Crouch and A.M. Starfield 1. Boundary element methods in solid mechanics. Allen and Unwin, London, 1983.

E.M. Din'kin and B.P. Osilenker 1. Weighted estimates of singular integrals and their applications. (Russian) Mathematical analysis, v. 21 (Russian) 42-129; Itogi nauki i tekhniki, Akad. Nauk SSSR, Vsesoyuzn. Inst. Nauchn. i Tekhn. Inform., Moscow, 1983.

R.V. Duduchava 1. Integral equations with fixed singularities. Teubner, Leipzig, 1979.

2. General singular integral equations and the basic problems of the plane theory of elasticity. (Russian) Trudy Tbiliss. Mat. Inst. Razmadze 
82(1986), 45-89.

3. On multidimensional singular integral operators. I, II. J. Operator Theory 11(1984), 41-76, 199-214.

R.V. Duduchava and T.I. Latsabidze 1. On the index of singular integral equations with complex-conjugate functions on piecewise-smooth lines. (Russian) Trudy Tbiliss. Mat. Inst. Razmadze 76(1985), No. 82, 40-59.

R.V. Duduchava, D.G. Natroshvili and E.M. Shargorodsky 1. Boundary value problems of the mathematical theory of cracks. (Russian) Proc. I. Vekua Inst. Appl. Math. (Tbiliss. Gos. Univ. Inst. Prikl. Mat. Trudy) 39(1990), 68-84.

G.I. Eskin 1. Boundary value problems for elliptic pseudodifferential equations. Transl. Math. Monographs, AMS, 52, Providence, Rhode Island, 1981.

T.G. Gegelia and R.K. Chichinadze 1. Solution in quadratures of the basic problem of thermoelasticity for a sphere and a spherical cavity. $Z$. Anal. Anwendungen 8(1989), No. 6, 515-553.

2. Boundary value problems of mechanics of continuum media for a sphere. 1-243 (to appear).

T.G. Gegelia and L. Jentsch 1. Uniqueness theorems in linear theory of micropolar solids. Z. Anal. Anwendungen (to appear).

I.Ts. Gokhberg 1. To the theory of multidimensional singular equations. (Russian) Dokl. Akad. Nauk SSSR 133(1960), No. 6, 1279-1282.

A.E. Green and P.N. Naghdi 1. On thermodynamics and nature of the second law for mixtures of interacting continua. Quart. J. Mech. and Appl. Math. 31(1978), part 3, 265-293.

A.E. Green and T.R. Steel 1. Constitutive equations for interacting continua. Internat. J. Engrg. Sci. 4(1966), No. 4, 483-500.

E. Hellinger and O. Toeplitz 1. Integralgleichungen und Gleichungen mit unendlich vielen Unbekannten. Encykl. Math. Wiss. II C 13, Teubner, Leipzig, 1928.

L. Hörmander 1. Pseudo-differential operators. Comm. Pure Appl. Math. 18 (1965), No. 3, 501-517.

L. Jentsch 1. Bemerkungen zu einigen gekoppelten Randwertproblemen der Thermoelastostatik. Wiss. Z. Karl-Marx-Univ. Leipzig Math.Natur. Reihe 25(1976), No.1, 39-52.

2. Über stationäre thermoelastische Schwingungen in inhomogenen Körpern. Math. Nachr. 64(1974), 171-231.

3. Stationäre thermoelastische Schwingungen in stückweise homogenen Körpern infolge zeitlich periodischer Aussentemperatur. Math. Nachrichten 69(1975), 15-37.

4. Zur numerischen Behandlung von gekoppelten Randwertproblemen der Thermoelastostatik. Wiss. Z. der Karl-Marx-Universität Leipzig, Math.Natur. Reihe 25(1976), No. 1, 27-38. 
5. Zur Existenz von regulären Lösungen der Elastostatik stückweise homogener Körper mit neuen Kontaktbedingungen an den Trennflächen zwischen zwei homogen Teilen. Abh. der Sächs. Akad. Wiss. Leipzig Math.Natur. Kl. 53(1977), No. 2, 1-45.

6. Die Greensche Matrix für zwei aneinander reibungsfrei gleitende Halbräume mit verschiedenen Lamé'schen Moduln. Z. angewandte Math. Mech. 58 (1978), 209-224.

7. Die elastostatischen Greenschen Tensoren 1. und 4. Art für den Halbraum als Grenzfälle eines Tensors für zwei aneinandergrenzende Halbräume mit verschiedenen Lamé'schen Moduln. Sem. I.Vekua Inst. Appl. Math. Tbilisi St. Univ. Reports (Sem. Inst. Prikl. Mat. Dokl. 12-13(1978), 49-66.

8. Thermoelastostatik stückweise homogener Körper mit gleitenden Einschlüssen. Demonstratio Math. XII (1979), No. 1, 261-280.

9. Probleme der mathematischen Elastizitätstheorie stückweise homogener Körper, 7. Tagung über Probleme und Methoden der Mathematischen Physik, Technishe Hochschule Karl-Marx-Stadt (Chemnitz), 1979, Tagungsberichte, 24-35.

10. Zur Theorie der Wärmespannungen in Bimetallkörpern, Wiss. Z. Karl-Marx-Univ. Leipzig, Math.-Natur. Reihe, 29(1980), No. 1, 49-58.

11. Über einige neuere Probleme der Elastizitätstheorie stückweise homogener Körper. FMC-Series, Akademie der Wissenschaften, Institut für Mechanik, Berlin und Karl-Marx-Stadt (Chemnitz) (1982), No. 2, 79-107.

12. Über Randintegralmethoden bei Randkontaktaufgaben, 9. TMP, Proceedings, TU Chemnitz, 1988, Teubner-Texte zur Mathematik, Bd. 111, 110-119.

13. On a thermal stress problem for contacting half-spaces with inclusions of other material involving a new method for computing potentials and singular integrals. Math. Methods Appl. Sci. 10(1988), 225-243.

14. Zur klassischen Kontaktaufgabe der Elastostatik. Z. Anal. Anwendungen 9(1990), No. 5, 433-453.

15. On a new approach for the contact problem of thermoelastostatics. Reports Enlarg. Session Sem. I. Vekua Inst. Appl. Math. (Dokl. Rassh. Zased. Sem. Inst. Prikl. Mat. Tbiliss. Gos. Univ. 4(1989), No. 2, 107-110.

16. Green's contact functions for heat conduction of two half-spaces and half-planes with application to several boundary contact problems. Math. Methods Appl. Sci. 4(1991), 633-661.

17. On boundary integral methods for contact problems. In Proceedings of the Symposium on Continuum Mechanics and Related Problems of Analysis, Tbilisi, June 5-12, 1991., 8p, Metsniereba, Tbilisi (to appear).

18. Über ein Bimetallproblem in der Ebene. Z. Anal. Anwendungen 1(1982), No. 5, 67-92. 
19. Bimetallprobleme mit verschiedenen Kontaktbedingungen, 8. Tagung über Probleme und Methoden der Mathematischen Physik, Technische Hochschule Karl-Marx-Stadt (Chemnitz), 1983, Teubner-Texte zur Mathematik, Bd. $63,111-121$.

20. Über ein Bimetallproblem der Ebene mit der Kontaktbedingung des reibungsfreien Gleitens. Proc. Tbilisi Univ. (Trudy Tbiliss. Univ. Mat. Mekh. Astronom.) 257(1985), 83-102.

21. Der Greensche Kontakttensor der Elastostatik für zwei fest verbundene Halbebenen. Z. angewandte Math. Mech. 61(1981), 343-344.

22. Über ebene Bimetallprobleme mit verschiedenen Kontaktbedingungen und Dirichletschen Randbedingungen. Z. Anal. Anwendungen 4(1985), No. 5, 437-453.

23. Über ebene Bimetallprobleme für das Aussengebiet mit verschiedenen Rand- und Kontaktbedingungen. Z. Anal. Anwendungen 5(1986), No. 5, 385-397.

24. Über ein ebenes Bimmetallproblem mit einen speziellen Rissbildung. Z. angewandte Math. Mech. 66(1986), 351-361.

25. On the boundary transmission problem of thermoelastostatics in a plane domain with interface corners. Preprint 93-1, DFG-Schwerpunkt Randelementmethoden. To appear in Math. Methods Appl. Sci.

L. Jentsch and J. Maul 1. Zur Elastizitäts- und Thermoelastizitätstheorie - Potentialtheoretische Methoden in Anwendung auf verschiedene Modelle. Math. Research, Bd. 4, Akademie-Verlag, Berlin, 1980, 1-155.

L. Jentsch and D. Natroshvili 1. Nonclassical interface problems for piecewise homogeneous anisotropic elastic bodies. Preprint 93-7, DFGSchwerpunkt Randelementmethoden. Submitted to Math. Methods Appl. Sci.

F. John 1. Plane waves and spherical means applied to partial differential equations. Interscience Publishers Ltd., London, 1955.

R.V. Kapanadze 1. Investigation of boundary value problems of the elasticity theory for anisotropic media by the potential method. (Russion) Trudy Tbiliss. Mat. Inst. Razmadze 87(1987), 82-113.

2. Singular integral operators on manifolds with boundary. Proc. Georgian Acad. Sci. Math. 1(1993), No. 4, 423-441.

L.P. Khoroshun and N.S. Soltanov 1. Thermoelasticity of two-component mixtures. (Russian) Naukova dumka, Kiev, 1984.

R.J. Knops and L.E. Payne 1. Uniqueness theorems in linear elasticity. Springer tracts in natural philosophy, v. 19, Springer-Verlag, BerlinHeidelberg-New York, 1971.

J.J. Kohn and L. Nirenberg 1. An algebra of pseudodifferential operators. Comm. Pure Appl. Math. 18(1965), Nos. 1, 2, 269-305.

G.V. Kolosov 1. On one application of the theory of functions of a complex variable to a plane problem of the mathematical theory of elasticity. 
(Russian) Yuryev, 1909.

2. Application of a complex variable to the theory of elasticity (Russian) Moscow, 1935.

V.A. Kondrat'ev and O.A. Oleinik 1. Korn's inequalities and the uniqueness of solutions of classical boundary value problems in unbounded domains for a system in elasticity theory. (Russian) Current problems in mathematical phisics. (Russian) (Proc. all-union symposium; Tbilisi, 1987), v. 1, 35-63, Tbilisi University Press, Tbilisi, 1987.

2. On Korn's inequalities. C. R. Acad. Sci. Paris Sér. I Math. 308(1989) No. 16, 483-487.

J. Král 1. Integral operators in potential theory. Lecture Notes. Math., v. 823 Springer, Berlin etc., 1980.

V.D. Kupradze, T.G. Gegelia, M.O. Basheleishvili and T.V. Burchuladze 1. Three-dimensional problems of the mathematical theory of elasticity and thermoelasticity. (Translated from Russian) North-Holland Publishing Company, Amsterdam-New-York-Oxford, 1979; Russian origonal: Nauka, Moscow, 1976.

D.A. Kveselava 1. The Hilbert boundary value problem and singular integral equations in the case of intersecting contours. (Russian) Trudy Tbiliss. Mat. Inst. Razmadze 17(1949), 1-27.

M.G. Kvinikadze 1. On some boundary-contact problems of dynamics of the elasticity theory an anisotropic nonhomogeneous elastic medium. (Russian) Sem. I. Vekua Inst. Appl. Math. Tbilisi St. Univ. Reports (Sem. Inst. Prikl. Mat. Dokl. 2(1986), 68-71.

2. Some oscillation boundary-contact problems of the elasticity theory for unbounded isotropic nonhomogeneous media. (Russian) Proc. Tbilisi Univ. (Trudy Tbisliss. Univ. Mat. Mekh. Astronom. 270(1987), 62-86.

3. Asymptotics for $t \rightarrow \infty$ of solutions of boundary-contact problems for hyperbolic systems. (Russian) Proc. Tbilisi Univ. (Trudy Tbisliss. Univ. Mat. Mekh. Astronom.) 90(1988), 115-126.

O.A. Ladyzhenskaya 1. Mathematical questions of dynamics of a viscous noncompressible fluid. (Russian) Moscow, 1970.

T.I. Latsabidze 1. Singular integral operators with a complex conjugation on piecewise-smooth lines. (Russian) Trudy Tbiliss. Mat. Inst. Razmadze 76(1985), 107-122.

L. Lichtenstein 1. Neuere Entwicklung der Potentialtheorie. Konforme Abbildung. Encykl. Math. Wiss., II C 3, 177-377, Teubner, Leipzig, 19091921.

A.T. Lonseth 1. Sources and applications of integral equations. SIAM Rev. 19(1977), No. 2, 241-278.

A.E.H. Love 1. A treatise on the mathematical theory of elasticity. 4th edition. Cambridge University Press, 1959. 
O.I. Maisaya and R.I. Gachechiladze 1. Investigation of noncorrect problems of the classical and couple-stress theory of elasticity. (Russian) Trudy Tbiliss. Mat. Inst. Razmadze 87(1987), 107-122.

2. Inversion method for contact problems of the elasticity theory. (Russian) Bull. Acad. Sci. Georgian SSR 126(1987), No. 3, 497-500.

R. Marcolongo 1. Teoria matematica dell equilibrio dei corpe elastici. Milan, 1904.

J. Maul 1. Eine einheitliche Methode zur Lösung der ebenen Aufgaben der linearen Elastostatik. Schriftenreihe des ZIMM bei der Akademie der Wissenschaften der DDR. Heft 24, Akademie-Verlag, Berlin, 1976.

2. Mixed contact problems in plane elasticity. Z. Anal. Anwendungen 2(1983), 207-234, 481-509.

V.G. Maz'ya 1. Boundary integral equations. (Russian) Current problems in mathematics. Fundamental directions, v. 27, (Russian) 131-229; Itogi nauki i tekhniki, Akad. Nauk SSSR, Vsesoyuzn. Inst. Nauchn. $i$ Tekhn. Inform., Moscow, 1988.

N.I. Muskhelishvili 1. Some basic problems of the mathematical theory of elasticity. (Russian) 5th edition, Fizmatgiz, Moscow, 1966; English translation from the third Russian edition of 1949 P. Noordhoff (Ltd.), Groningen, 1953.

2. Singular integral equations. (Russian) 3rd edition, Nauka, Moscow, 1968; English translation from the first Russian edition P. Noordhoff (Ltd.), Groningen, 1953.

N.I. Muskhelishvili and D.A. Kveselava 1. Singular integral equations with Cauchy-type kernels on open contours. (Russian) Trudy Tbiliss. Mat. Inst. Razmadze 11(1942), 141-172.

D.G. Natroshvili 1. Dynamic problems of theormoelasticity for anisotropic homogeneous media. (Russian) Differentsial'nye Uravneniya 20(1984), No. 1, 87-98.

D.G. Natroshvili, O.O. Chkadua, and E.M. Shargorodsky 1. Mixed problems for homogeneous anisotropic bodies. (Russian) Proc. I. Vekua Inst. Appl. Math. (Tbiliss. Gos. Univ. Inst. Prikl. Mat. Trudy) 39(1990), 139-180.

D.G. Natroshvili, A. Ya. Jagmaidze and M.Zh. Svanadze 1. Some problems of the linear theory of elastic mixtures. (Russian) Tbilisi University Press, 1986.

D.G. Natroshvili and E.M. Shargorodsky 1. Three-dimensional problems of dynamics for elastic bodies with cuts. (Russian) Trudy Tbiliss. Mat. Inst. Razmadze 140(1990), No. 2, 281-284.

H. Nicolesco 1. Les founctions polyharmoniques. Ed. Hermann, Paris, 1936.

K.G. Odqvist 1. Über die Randwertaufgaben der Hydrodynamik zäher Flüssig-veiten. Math. Z. 32(1930), 329-375. 
S. Prössdorf 1. Einiqe Klassen singulären Gleichungen. AkademieVerlag, Berlin, 1974.

2. Linear integral equations. (Russian) Current problems in mathematics. Fundamental directions, v. 27, (Russian) 5-131; Itogi nauki i tekhniki, Akad. Nauk SSSR, Vsesoyuzn. Inst. Nauchn. i Tekhn. Inform., Moscow, 1988.

S. Rempel and B.-W. Schulze 1. Index theory of elliptic boundary problems. Akademie-Verlag, Berlin, 1982.

R.T. Seeley 1. Singular integrals on compact manifolds. Amer. J. Math. 81(1959), No. 4, 658-690.

2. Regularization of singular integral operators on compact manifolds. Amer. J. Math. 83(1961), No. 2, 265-275.

E. Shamir 1. Elliptic systems of singular integral operators. I: The half-space case. Trans. Amer. Math. Soc. 127(1967), 107-124.

E.M. Shargorodsky 1. Boundary value problems for elliptic pseudodifferential operators: the half-space case. (Russian) Trudy Tbiliss. Mat. Inst. Razmadze 99, 44-80 (to appear).

2. Boundary value problems for elliptic pseudodifferential operators on manifolds. (Russian) Trudy Tbiliss. Mat. Inst. Razmadze 105, 108-132 (to appear).

S.L. Sobolev 1. Some applications of functional analysis in mathematical physics. (Russian) Leningrad University Press, Leningrad, 1950; Second edition: Nauka, Moscow, 1988.

A.P. Soldatov 1. One-dimensional singular operators and boundary value problems of the theory of functions. (Russian) . Vysshaya Shkola, Moscow, 1991.

V.S. Sologub 1. Development of the theory of elliptic equations in the 18th and 19th centuries. (Russian) Naukova Dumka, Kiev, 1975.

T.R. Steel 1. Applications of a theory of interacting continua. Quart. J. Mech. Appl. Math. 20(1967), No. 1, 57-72.

O. Tedone 1. Allgemeine Theoreme der mathematischen Elastizitätslehre. Encykl. Math. Wiss. IV 24, Teubner, Leipzig, 1907-1914.

H. Tiersten and M. Jahanmir 1. A theory of composites modelled as interpenetrating solid continua. Arch. Rational Mech. Anal. 65(1977), 153-192.

E. Trefftz 1. Mechanik der elastischen Körper. Handbuch der Physik, VI, Springer-Verlag, Berlin, 1928.

F. Treves 1. Introduction to pseudodifferential and Fourier integral operators. 1,2. Plenum Press, New York, 1982.

H. Triebel 1. Interpolation theory, function spaces, differential operators. VEB Deutsch. Verl. Wiss., Berlin, 1978.

2. Theory of function spaces. Birkhäuser Verlag, Basel-Boston-Stuttgart, 1983. 
C. Truesdell and R. Toupin 1. The classical field theories. Handbuch der Physik. III/1, Berlin, Springer, 1960.

B.R. Vainberg 1. The radiation, limiting absorption and limiting amplitude principles in the general theory of partial differential equations. (Russian) Uspekhi Mat. Nauk 3(129)(1966), 115-194.

N.P. Vekua 1. Systems of singular integral equations. (Russian) Fizmatgiz, Moscow, 1970; English translation from the first Russian edition P. Noordhoff (Ltd.), Groningen, 1967.

P. Vilaggio 1. Kelvin's solution and nuclei of strain in a solid mixture. Ann. Scuola Norm. Sup. Pisa. Serie 4, 10 (1983),?? No. 1, 107-124.

I.I. Vorovich, V.M. Aleksandrov and V.A. Babeshko 1. Nonclassical mixed problems of the theory of elasticity. (Russian) Moscow, 1974.

W.L. Wendland 1. Elliptic Systems in the Plane. Pitman, London, 1979.

N. Wiener and E. Hopf 1. Über eine Klasse singulärer Integralgleichungen. Sitzungsber. Preuss. Akad. d. Wiss, 1931, 696-706.

Sh.P. Zazashvili 1. On one mixed problem of the bending of an anisotropic plate. (Russian) Proc. I.Vekua Inst. Appl. Math. (Tbiliss. Gos. Univ. Inst. Prikl. Mat. Trudy) 23(1988), 49-65.

2. A mixed boundary value problem for an infinite anisotropic plate with an elliptic hole. (Russian) Sem. I. Vekua Inst. Appl. Math. Tbilisi St. Univ. Reports (Sem. Inst. Prikl. Mat. Dokl.) 3(1988), No. 1, 68-71.

3. A mixed boundary value problem for an infinite anisotropic plate with a rectilinear cut. (Russian) Sem. I.Vekua Inst. Appl. Math. Tbilisi Gos. Univ. Reports (Sem. Inst. Prikl. Mat. Dokl.) 4(1989), No. 2, 95-98.

4. The contact problems for two anisotropic half-planes with slits. Proc. Georgian Acad. Sci. Math. 1(1993), No. 3, 365-382.

(Received 12.11.1993)

Authors' addresses:

A. Razmadze Mathematical Institute Technische Universität Chemnitz Georgian Academy of Sciences Chemnitz, Germany

1, Z. Rukhadze St., Tbilisi, 380093

Republic of Georgia 